ARTICLE

Received 25 May 2016 | Accepted 14 Nov 2016 | Published 11 Jan 2017

DOI: $10.1038 /$ ncomms13944

OPEN

\title{
The mito-DAMP cardiolipin blocks IL-10 production causing persistent inflammation during bacterial pneumonia
}

Krishnendu Chakraborty ${ }^{1}$, Mahesh Raundhal ${ }^{1,2}$, Bill B. Chen ${ }^{1}$, Christina Morse ${ }^{1}$, Yulia Y. Tyurina ${ }^{3}$, Anupriya Khare ${ }^{1}$, Timothy B. Oriss ${ }^{1}$, Rachael Huff ${ }^{1}$, Janet S. Lee ${ }^{1}$, Claudette M. St. Croix ${ }^{4}$, Simon Watkins ${ }^{4}$, Rama K. Mallampalli', Valerian E. Kagan ${ }^{3}$, Anuradha Ray ${ }^{1,2}$ \& Prabir Ray ${ }^{1,2}$

Bacterial pneumonia is a significant healthcare burden worldwide. Failure to resolve inflammation after infection precipitates lung injury and an increase in morbidity and mortality. Gram-negative bacteria are common in pneumonia and increased levels of the mito-damageassociated molecular pattern (DAMP) cardiolipin can be detected in the lungs. Here we show that mice infected with Klebsiella pneumoniae develop lung injury with accumulation of cardiolipin. Cardiolipin inhibits resolution of inflammation by suppressing production of antiinflammatory IL-10 by lung CD11b ${ }^{+}$Ly $6 G^{\text {int }}$ Ly6C ${ }^{l}$ F $4 / 80^{+}$cells. Cardiolipin induces PPAR $\gamma$ SUMOylation, which causes recruitment of a repressive NCOR/HDAC3 complex to the IL-10 promoter, but not the TNF promoter, thereby tipping the balance towards inflammation rather than resolution. Inhibition of HDAC activity by sodium butyrate enhances recruitment of acetylated histone 3 to the IL-10 promoter and increases the concentration of IL-10 in the lungs. These findings identify a mechanism of persistent inflammation during pneumonia and indicate the potential of HDAC inhibition as a therapy.

\footnotetext{
${ }^{1}$ Pulmonary, Allergy and Critical Care Medicine, Department of Medicine, University of Pittsburgh School of Medicine, 3459 Fifth Avenue, Pittsburgh, Pennsylvania 15213, USA. ${ }^{2}$ Department of Immunology, 200 Lothrop St, University of Pittsburgh School of Medicine, E1040 BSTWR, Pittsburgh, Pennsylvania 15261, USA. ${ }^{3}$ Center for Free Radical and Antioxidant Health, Department of Environmental and Occupational Health, University of Pittsburgh School of Medicine, Bridgeside Point, 100 Technology Drive, Suite 350, Pittsburgh, Pennsylvania 15219, USA. ${ }^{4}$ Center for Biologic Imaging, University of Pittsburgh School of Medicine, 3500 Terrace St, Pittsburgh, Pennsylvania 15261, USA. Correspondence and requests for materials should be addressed to P.R. (email: rayp@pitt.edu).
} 
B acterial infection of the lung induces an inflammatory response, which is a natural useful response to eliminate the invading pathogen. However, unless terminated quickly, this inflammatory response can also precipitate pneumonia, which fails to resolve in some cases, resulting in a condition termed non-resolving pneumonia, which can cause acute lung injury ${ }^{1,2}$. Bacterial pneumonia continues to be a leading cause of morbidity and mortality worldwide ${ }^{3}$. Therefore, one of the pressing questions in medicine is how excess inflammation can be turned off. Resolution of inflammation is no longer considered a passive process but one that involves timely elaboration of different mediators that helps to terminate the inflammatory response and restore tissue homeostasis ${ }^{4-6}$.

Alveolar macrophages and neutrophils are the major cell types that phagocytose and kill internalized lung bacteria ${ }^{7,8}$, with alveolar macrophages responding immediately and subsequent infiltration of neutrophils in response to chemotactic stimuli ${ }^{9}$. Neutrophils generate noxious products including reactive oxygen species and proteases that are not only harmful to pathogens but also to the host ${ }^{3,10-12}$. Therefore, once the pathogen is cleared, the immediate goal of the host is to mount an appropriate anti-inflammatory response to limit further neutrophil recruitment and to clear apoptotic neutrophils in a process called neutrophil efferocytosis. The Gram-negative bacterium Klebsiella pneumoniae is frequently detected in hospital-acquired pneumonia, particularly in chronically ill patients, and is the third most commonly isolated organism from intensive care units in the United States ${ }^{13}$. We previously showed that

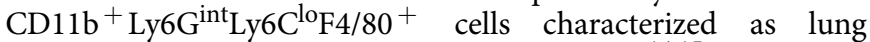
myeloid-derived suppressor cells (MDSCs) ${ }^{14,15}$ have an important function in neutrophil efferocytosis after infection with K. pneumoniae ${ }^{16}$.

High concentrations of the phospholipid cardiolipin have been detected in the lung fluid of patients with persistent pneumonia ${ }^{17}$. Cardiolipin is the principal lipid component of the inner mitochondrial membrane. Although cardiolipin is normally confined to mitochondria, during cell death it undergoes oxidation and is released into the extracellular milieu as a mitochondrial damage-associated molecular pattern (mito-DAMP) ${ }^{18-20}$. Here we show that cardiolipin induces SUMOylation of the nuclear receptor PPAR $\gamma$ at K107, which is distinct from the previously described SUMOylation at K395 induced by the PPAR $\gamma$ agonist rosiglitazone (rosi) ${ }^{21}$. Cardiolipin-induced SUMOylation inhibits interleukin (IL)-10 production, but does not interfere with tumour necrosis factor (TNF) production, the latter being inhibited by rosi-induced K395 SUMOylation. K107 SUMOylation in PPAR $\gamma$ causes recruitment of the repressive NCOR/HDAC3 complex to the IL-10 upstream regulatory region, but spares the TNF promoter. Exposure of MDSCs to extracellular cardiolipin causes accumulation of cyclic phosphatidic acid (cPA), which also induces PPAR $\gamma$ SUMOylation and recruitment of HDAC3 to the IL-10 promoter. Inhibition of HDAC activity in mice infected with $K$. pneumonia restores lung IL-10 levels and reduces TNF with gain in body weight, plus increases survival of the mice. Our study identifies cardiolipin as a mediator of non-resolving pneumonia via SUMOylation of PPAR $\gamma$ in which HDAC inhibition provides therapeutic benefit.

\section{Results}

Elevated oxidized cardiolipin level in infected lungs. We previously showed that mice infected with 100 colony-forming units (c.f.u.) of K. pneumoniae have a $100 \%$ survival rate while those infected with a higher dose (1,000 c.f.u.) show 50\% survi$\mathrm{val}^{16}$. When examined for the level of total oxidized phospholipids, a higher level was detected in the lung tissue of mice infected with 1,000 c.f.u. of $K$. pneumoniae as compared with that in mice infected with 100 c.f.u. (Fig. 1a). The level of extracellular host cardiolipin was found to be elevated in the lungs of mice infected with the higher bacterial dose $(1,000$ c.f.u.) when compared with uninfected controls (Fig. 1b,c). The level of bacterial cardiolipin was largely similar in the two groups. Moreover, the levels of oxidized forms (mono- and di-oxygenated) of the two most abundant C18:2-containing cardiolipin species were also higher in infected than in control samples (Fig. 1d).

We recently reported that concomitant exposure of cells to cardiolipin and lipopolysaccharide (LPS) in vitro inhibits the production of pro-inflammatory cytokines such as TNF- $\alpha$ by the cells ${ }^{22}$. However, these studies did not address the impact of cardiolipin in vivo on already established inflammation when cardiolipin levels increase in the extracellular milieu (Fig. 1a). Also, given that an increased level of the mito-DAMP cardiolipin was found in the lungs of pneumonia subjects ${ }^{17}$, we hypothesized that cardiolipin in an inflammatory niche has the ability to incite a condition of non-resolving lung inflammation. To test our hypothesis, a low dose of bacterial LPS was instilled in the lungs of mice intratracheally (i.t.). One hour later, cardiolipin was administered i.t. into half of these mice. Inflammation and lung leakage, both markers of pneumonia, were examined by assaying myeloperoxidase (MPO) activity, a measure of neutrophil numbers, and albumin level respectively in the bronchoalveolar lavage (BAL) fluid collected from the mice. While both MPO activity and lung albumin level initially increased in the BAL fluid of mice that received LPS alone as assessed on day 3 after treatment with LPS, both of these parameters were significantly lower on day 6 following LPS treatment (Fig. 2b,c). In the mice that received both LPS and cardiolipin, on day 3 posttreatment, both MPO activity and albumin level in the BAL fluid were higher than that in mice treated with LPS alone and the decrease in these measures was non-significant after day 6 in contrast to that observed in the LPS-treated group (Fig. 2b,c). Mice treated with LPS followed by cardiolipin showed significantly higher levels of the pro-inflammatory cytokines TNF and IL- 6 but reduced level of the anti-inflammatory cytokine IL-10 in their lungs as compared with those treated with LPS alone (Fig. 2c).

Lung tissue resident $\mathrm{CD}_{11 b^{+}} \mathrm{Ly}_{6 \mathrm{G}^{\mathrm{int}}} \mathrm{Ly} 6 \mathrm{C}^{\mathrm{lo}} \mathrm{F} 4 / 80^{+}$cells, resembling MDSCs, produce $\mathrm{IL}-10$ that serves to curtail inflammation during bacterial infection ${ }^{16,23}$. As shown in Supplementary Fig. 1, this cell is the major source of the net IL-10 produced in the lungs of mice after LPS treatment. Flow cytometric analysis of lung MDSCs from LPS + cardiolipintreated mice showed marked suppression of intracellular IL-10 compared with expression in the same cells isolated from mice treated with LPS alone (Fig. 2d). To test whether cardiolipin is actively taken up by lung MDSCs, LPS was delivered i.t. into mice and after $48 \mathrm{~h}$, fluorescently labelled cardiolipin was instilled i.t. into the mice. When examined by confocal microscopy, sorted MDSCs from the lungs of these mice showed uptake of cardiolipin (Supplementary Fig. 2a). Sorted MDSCs from LPS-treated mice also showed suppression of IL-10 when incubated ex vivo with both LPS plus cardiolipin (Supplementary Fig. 2b), suggesting a direct suppressive effect of cardiolipin on IL-10 production from MDSCs.

WT but not IL-10-deficient MDSCs resolve lung inflammation. Since our data suggested that cardiolipin-mediated suppression of IL-10 production from lung MDSCs leads to non-resolving lung inflammation, we wondered whether adoptive transfer of in vitro 
a

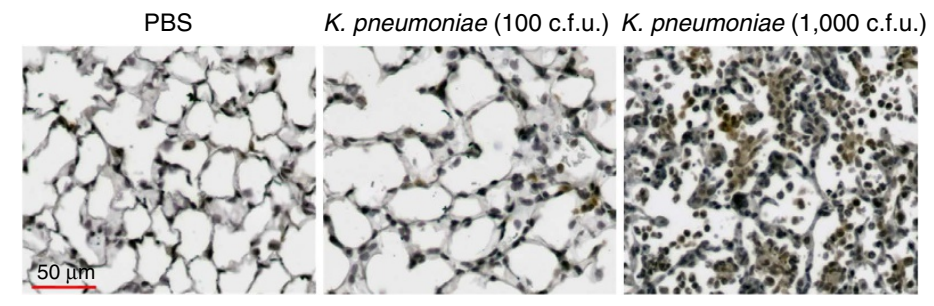

C

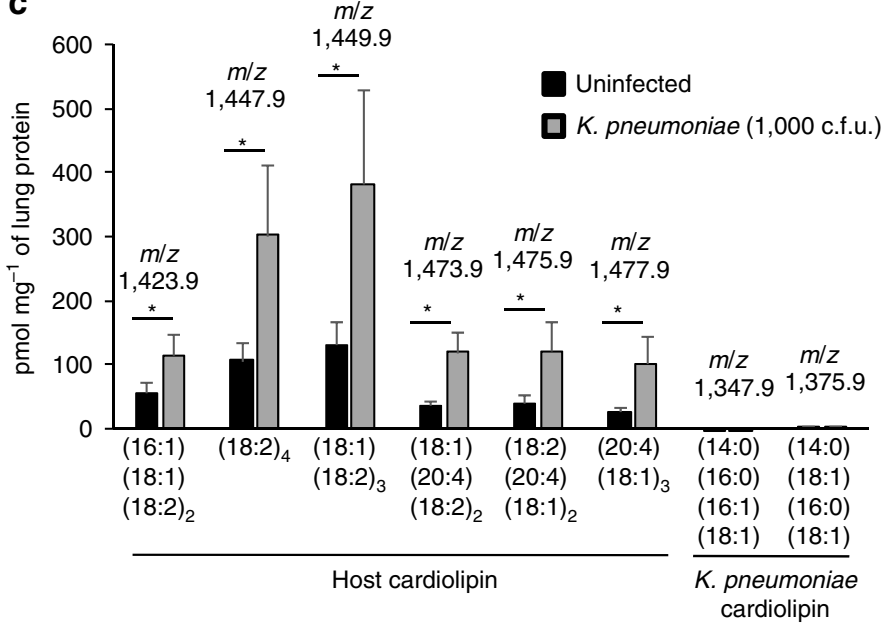

b

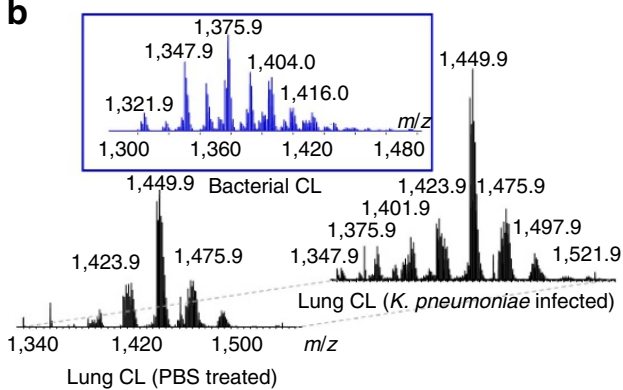

d

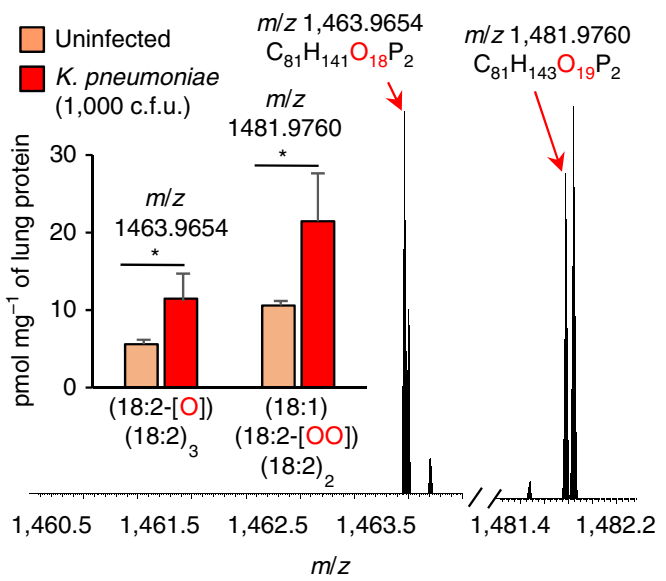

Figure 1 | Increased level of cardiolipin in lungs of mice infected with $\boldsymbol{K}$. pneumoniae. (a) C57BL/6J mice were either left uninfected or infected intratracheally (i.t.) with K.pneumoniae (100 or 1,000 c.f.u.). Day 3 post infection, lung tissue sections of mice were stained for oxidized phospholipids. (b) LC/MS spectra of host cardiolipin obtained from lungs of PBS-treated mice (left) and infected mice (right). Middle panel depicts LC/MS spectrum of bacterial cardiolipin. (c) Quantitation of major host and bacterial cardiolipin molecular species from spectral data shown in b. (d) LC-MS spectra of mono- $(\mathrm{m} / \mathrm{z}$ 1,463.9654) and di- $(\mathrm{m} / \mathrm{z}$ 1,481.9760) oxygenated cardiolipin molecular species and their quantitative assessments in the lung. Data shown are mean \pm s.d. and all data are representative of two independent experiments with $n=3$ mice per group. Significance was calculated using Student's unpaired two-tailed $t$ test for comparisons involving two groups or one-way ANOVA with Tukey's post hoc test for multiple pairwise comparisons. ${ }^{\star} P<0.05$. $\mathrm{CL}$, cardiolipin.

generated MDSCs would minimize lung inflammation and restore tissue homeostasis in mice treated with both LPS and cardiolipin. To this end, bone marrow cells were cultured with GM-CSF and LPS for nine consecutive days. CD11b ${ }^{+}$Ly6G $^{\text {int }} \mathrm{F} 4 /$ $80^{+}$cells producing a high level of IL-10 were sorted ${ }^{14,16}$ (Supplementary Fig. 3) and adoptively transferred into mice that had received both LPS and cardiolipin the day before. Change in body weight was then monitored in all mice. Although there was an initial loss of body weight in mice that received LPS alone, the mice regained their weight from day 3 onwards. However, the mice treated with both LPS and cardiolipin continued to lose weight until day 6 when they needed to be euthanized (Fig. 2e). Mice that received phosphate-buffered saline (PBS) or cardiolipin alone showed minimal or no change in body weight (Fig. 2e). Mice that received MDSCs generated from bone marrow cells of wild-type (WT) mice showed a significant gain of their initial body weight (Fig. 2e) along with reduced cellular infiltration in the lung on day 6 after LPS + cardiolipin treatment as compared with LPS + cardiolipin-treated mice that did not receive the cells (Fig. 2f). Mice that received the MDSCs also showed decreased MPO activity and protein leak on day 6 following LPS + cardiolipin treatment (Fig. 2g,h). However, MDSCs generated from $I l 10^{-1}-$ mice failed to mitigate any of the inflammation parameters (Fig. 2e-h). The IL-10 level was higher in the lungs of LPS + cardiolipin-treated mice that received MDSCs generated from WT mice as compared with those that received cells generated from $\mathrm{IllO}^{-/-}$mice (Fig. 2i).
Thus, overall, the mice that received WT MDSCs displayed improvement in all outcome measures compared with those that received IL-10-deficient MDSCs. These data further strengthened our hypothesis that IL-10 from MDSCs is a critical factor for resolution of lung inflammation following bacterial infection as also shown in our earlier study ${ }^{16}$. However, given that the IL-10-deficient MDSCs had a small inhibitory effect on MPO activity and BAL albumin levels on day 6 , it is likely that other mediators produced by these cells also contribute to resolution of inflammation.

PPAR $\gamma$ mediates LPS-induced IL-10 production in lung MDSCs. We next explored the mechanism underlying suppression of IL-10 production from lung MDSCs in the presence of cardiolipin. Different transcription factors have been associated with regulation of IL-10 gene expression in various cell types ${ }^{24}$. Although IL-10 was previously shown by us to be an important mediator of lung MDSC function ${ }^{14,16,23}$, the regulatory elements in the IL-10 promoter that control its expression in the lung MDSCs were not elucidated. Given that the focus of our study was lung inflammation and its resolution, using Ingenuity Knowledge Base (Qiagen) we first identified 718 molecules that have been previously associated with lung inflammation. Since our data suggested that cardiolipin transcriptionally modulates IL-10 expression, we applied the 'build tool' of Ingenuity Pathway Analysis (IPA) (Qiagen) to reduce the list of 
718 molecules to include only transcription factors, liganddependent nuclear receptors and cytokines. Since cardiolipin is a lipid molecule, we next used IPA's 'path explorer' tool to explore the factors involved in lipid-mediated regulation of IL-10 gene expression. This search identified $\operatorname{PPAR} \gamma$ as the only transcription factor associated with IL-10 production. Analysis of the upstream regulatory region (URR) in the IL-10 gene also revealed multiple PPAR $\gamma$ response elements (PPRE) (Fig. 3a).
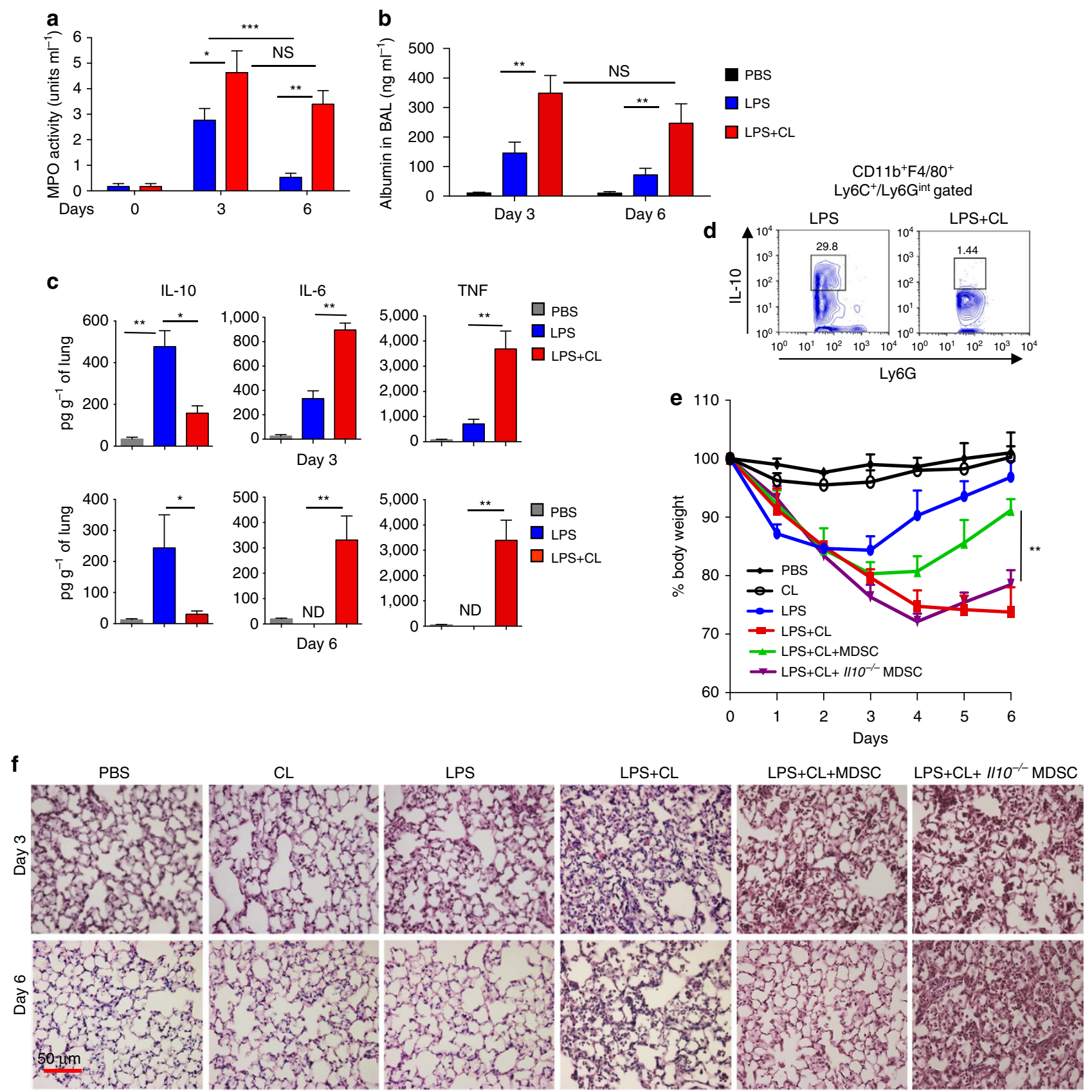

$\begin{aligned} \mathbf{a} & \square \mathrm{LPS}+\mathrm{CL}+\mathrm{MDSC} \\ & \square \mathrm{LPS}+\mathrm{CL}+1110^{--} \mathrm{MDSC}\end{aligned}$

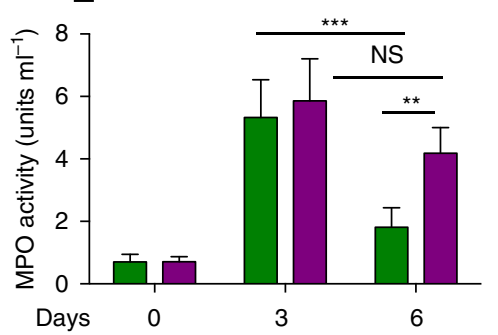

Days 0 h

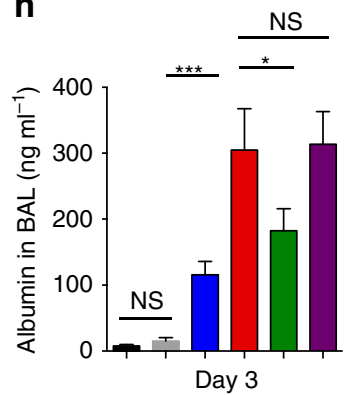

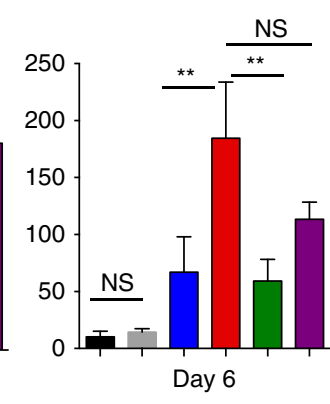

i

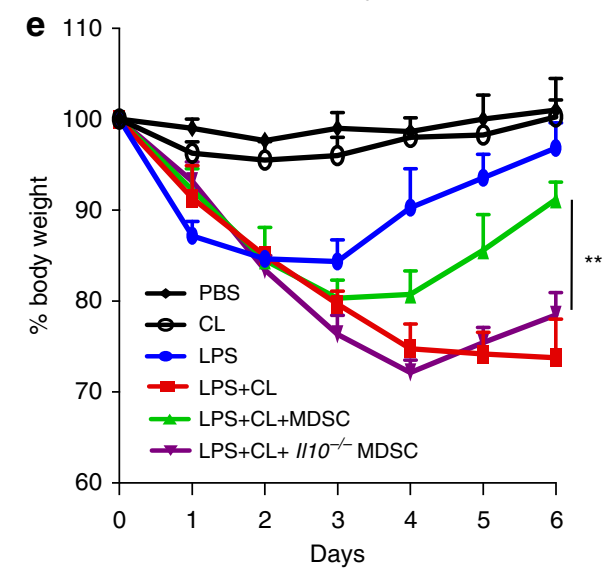

$\mathrm{CD} 11 \mathrm{~b}^{+} \mathrm{F} 4 / 80^{+}$

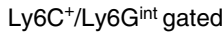

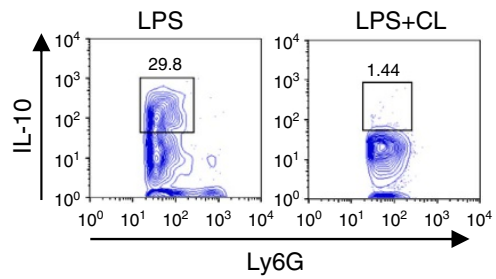


Indeed, treatment of lung MDSCs with rosi promoted IL-10 production from the cells (Supplementary Fig. 4a). In published literature, phospholipids have been shown to function as endogenous regulators of $\operatorname{PPAR} \gamma$ activity ${ }^{25,26}$. As shown in Fig. 3b, use of the specific inhibitor of PPAR $\gamma$, GW9662, suppressed IL-10 mRNA and protein levels in the lung MDSCs to the same degree as cardiolipin. To be certain that GW9662 specifically inhibits PPAR $\gamma$ activity, we performed a PPREluciferase reporter assay in RAW 264.7 cells transfected with both PPRE-luciferase reporter construct and a PPAR $\gamma$ expression vector. Transfected cells were treated with either rosi or LPS alone or in combination with GW9662. We observed a significant reduction in reporter activity with GW9662 in both rosi- and LPS-treated cells suggesting specific inhibition of PPAR $\gamma$ activity with GW9662 (Fig. 3c). In a similar fashion, cardiolipin also inhibited reporter activity induced by a combination of rosi and LPS (Supplementary Fig. 4b). Next we studied the effect of GW9662 on IL-10 promoter activity to investigate whether PPAR $\gamma$ is involved in regulating IL-10 production post-LPS stimulation. To this end, we transfected RAW 264.7 cells with an IL-10 reporter construct along with PPAR $\gamma$ expression vector. We observed a marked reduction in IL-10 reporter activity in the cells treated with both LPS and GW9662 as compared with cells treated with LPS alone, suggesting involvement of PPAR $\gamma$ in LPS-induced IL-10 (Fig. 3d). Also, knocking down PPAR $\gamma$ in lung MDSC cells by PPAR $\gamma$-specific siRNA (Supplementary Fig. 4c) significantly inhibited LPS-induced IL-10 production, while TNF expression remained unchanged (Fig. 3e). IL-10 reporter assay with WT (extending to $-1,365 \mathrm{bp}$ in the URR) and mutant reporter constructs containing deletions of individual PPREs within the context of the full length promoter revealed that the PPRE at the -339 bp location was critical for optimal IL-10 promoter activation (Fig. 3f). Collectively, these data suggested an important role for PPAR $\gamma$ in IL-10 gene expression in lung MDSCs as well as in peritoneal macrophages. These data were in line with findings in previous in vivo studies where PPAR $\gamma$ activation by a synthetic ligand ${ }^{27}$ or by a specific gut bacterium ${ }^{28}$ increased IL-10 production although the regulatory mechanism was not explored in these studies.

To directly demonstrate recruitment of PPAR $\gamma$ to the IL-10 URR in lung MDSCs, we performed a chromatin immunoprecipitation (ChIP) experiment. Although a moderate increase in enrichment of PPAR $\gamma$ was observed in LPS-treated samples, both untreated as well as LPS + cardiolipin-treated samples also showed significant binding of PPAR $\gamma$ to the IL-10 URR (Fig. 4a, left panel). The quantitative analysis of two independent ChIP experiments was performed by qPCR providing similar information (Fig. 4a, right panel). This observation led us to hypothesize that under unstimulated and cardiolipin-treated conditions, PPAR $\gamma$ is recruited to the IL-10 URR in association with corepressors such as NCOR along with HDAC3 (a histone deacetylase). If this were true, while PPAR $\gamma$ would remain bound to the PPREs under these conditions, it would not be functionally active (Fig. 4b). To verify our hypothesis, we first performed immunoprecipitation (IP) experiments using antibodies against NCOR or HDAC3 followed by western blot analysis using anti-PPAR $\gamma$ antibody. Indeed, under unstimulated condition, PPAR $\gamma$ co-precipitated with NCOR and HDAC3 (Fig. 4c). We also performed the reverse experiment using anti-PPAR $\gamma$ for IP followed by western blot analysis using antibodies against NCOR and HDAC3. This association between PPAR $\gamma$ and NCOR/HDAC3 was, however, abolished upon LPS treatment and was restored when cells were treated with LPS + cardiolipin (Fig. 4c). The results of these experiments demonstrated complex formation between PPAR $\gamma$ and NCOR/HDAC 3 under conditions where IL-10 expression was low, which were under basal conditions or in the presence of LPS + cardiolipin. To further test our hypothesis, we examined recruitment of NCOR or HDAC3 to the IL-10 URR under these conditions, for which ChIP assay was performed. Recruitment of NCOR and HDAC3 to the IL-10 URR was observed under both basal and LPS + cardiolipin-treated conditions, which was not the case when cells were treated with LPS alone (Fig. 4d). The opposite was true for the recruitment of the coactivator p300 to the IL-10 URR (Fig. 4d). Taken together, these observations showed that cardiolipin prevents LPS-induced dissociation of the corepressor molecule NCOR and of HDAC3 from PPAR $\gamma$ associated protein complex on the IL-10 URR.

SUMOylation of PPAR $\gamma$ by cardiolipin blocks IL-10 production. Since SUMOylation of PPAR $\gamma$ has been shown to promote and stabilize corepressor/HDAC-associated PPAR $\gamma$ protein complex by inhibiting proteasome-mediated degradation ${ }^{21}$, we were next interested in determining whether cardiolipin has the ability to induce SUMOylation of PPAR $\gamma$. A marked increase in SUMOylation of PPAR $\gamma$ was observed in lung MDSCs treated with LPS + cardiolipin, which was largely diminished by the addition of the SUMOylation inhibitor ginkgolic acid (Fig. 5a, upper panel). SUMOylation of PPAR $\gamma$, however, was not detectable in untreated or LPS alone treated samples. We observed that increased PPAR $\gamma$ SUMOylation was associated with decreased IL-10 expression and inhibition of SUMOylation could overcome the cardiolipin-mediated suppression of IL-10 production (Fig. 5a, lower panel). SUMOylation of PPAR $\gamma$ by its ligand, rosi, has been shown to be critical for suppression of

Figure 2 | Induction of non-resolving lung inflammation with cardiolipin and low dose of LPS. (a,b) C57BL/6J mice were treated intratracheally (i.t.) with LPS $(20 \mu \mathrm{g}$ per mouse), either alone or in combination with cardiolipin $(100 \mu \mathrm{g}$ per mouse). Cardiolipin was administered $1 \mathrm{~h}$ post LPS instillation. PBS-treated mice were used as controls. On days 3 and 6 post-treatment, the mice were analysed for (a) myeloperoxidase activity (MPO) activity in BAL fluid, (b) BAL albumin levels, a measure of microvascular permeability and (c) levels of cytokines in lung homogenates measured using magnetic bead-based assay. (d) Mice were treated with LPS \pm cardiolipin for three consecutive days. Day 6 post-treatment, IL-10 expression in

CD11b ${ }^{+}$Ly6C ${ }^{l o}$ y6Gint $44 / 80+$ lung MDSCs was assayed by flow cytometry. Gating is shown in Supplementary Fig. 1. (e-h) Mice were treated with LPS either alone or in combination with cardiolipin. Twenty-four hour post-treatment, $1 \times 10^{6}$ bone marrow-derived MDSCs from wild type or $/ 110^{-/-}$mice were adoptively transferred i.t. into mice that had received both LPS and cardiolipin and the following parameters were studied day 3 and 6 post LPS \pm cardiolipin administration: (e) Change in body weight monitored over six days (presented as \% change from initial body weight). (f) lung pathology by H\&E staining of lung tissue sections, (g) MPO activity in BAL fluid and (h) BAL albumin level. (i) Level of IL-10 in lung homogenates measured 48 h post adoptive transfer. The experiment was initiated with the following numbers of mice: 3 for PBS, cardiolipin and LPS groups, 8 for LPS + cardiolipin, 7 for LPS + cardiolipin + MDSC (WT) and 8 for LPS + cardiolipin + MDSC (I/10-/-) groups. At the conclusion of the experiment on day 6,3 animals in PBS, cardiolipin, LPS and LPS + cardiolipin groups, 5 mice in the LPS + cardiolipin + MDSC (WT) group and 3 mice in the LPS cardiolipin + MDSC (II10-/-) were available for analysis. Statistical significance was calculated using one-way ANOVA or two-way ANOVA with Bonferroni's post hoc test was used for multiple pairwise comparisons. Data shown are mean \pm s.d. and data are representative of two independent experiments. ${ }^{\star} P \leq 0.05$, ${ }^{\star \star} P \leq 0.01$,

${ }^{\star \star \star} P \leq 0.001 ; \mathrm{NS}$, not significant. 
a

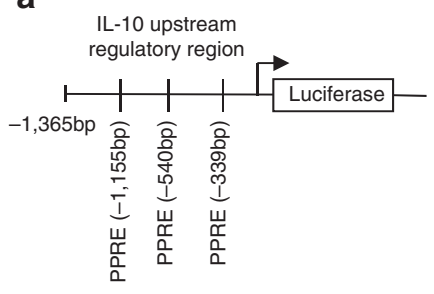

b

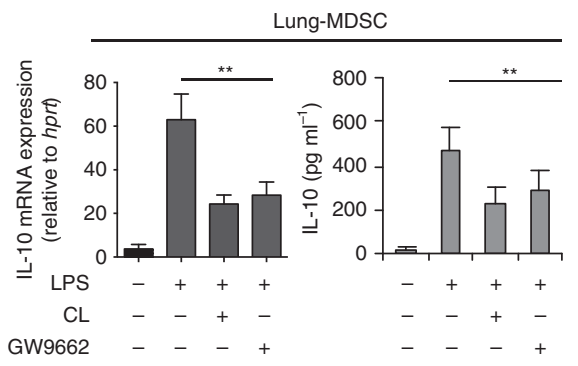

C

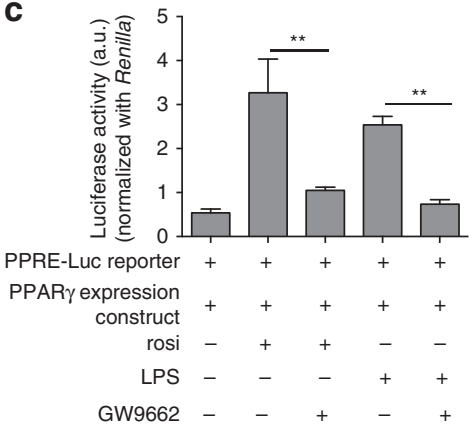

d

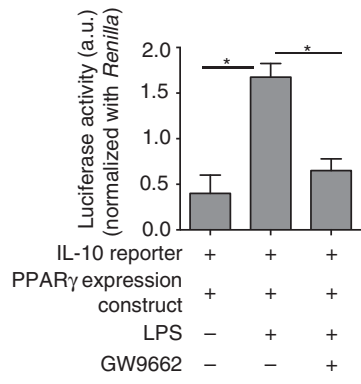

e

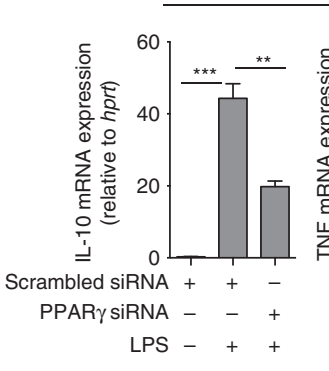

Lung-MDSC

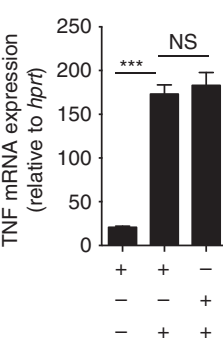

f

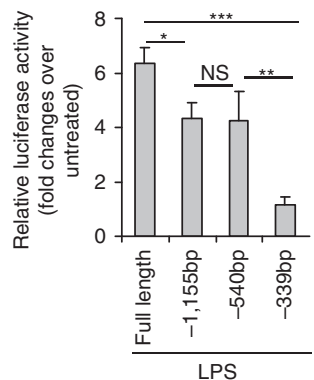

Figure 3 | PPAR $\gamma$ is critical for IL-10 regulation in lung MDSCs. (a) Schematic of IL-10 upstream regulatory region showing multiple PPAR/RXR response elements (PPAR-RE). (b) Lung MDSCs were treated ex vivo with LPS $\left(1 \mu \mathrm{g} \mathrm{ml}^{-1}\right)$ either alone or in combination with cardiolpin $\left(10 \mu \mathrm{g} \mathrm{ml}{ }^{-1}\right) \pm \mathrm{GW} 9662$ $(10 \mu \mathrm{M})$ for $6 \mathrm{~h}$, cardiolipin being added $1 \mathrm{~h}$ post LPS treatment. Levels of IL-10 mRNA and secreted IL-10 in the culture supernatant were measured by qPCR (left panel) and ELISA (right panel) respectively. Addition of cardiolipin alone did not promote IL-10 gene expression (data not shown). (c) RAW 264.7 cells were transfected with both PPRE-Luciferase reporter construct and PPAR $\gamma$ expression vector. Twenty-four hour post-transfection, cells were stimulated with rosiglitazone (rosi) or LPS \pm GW9662. Two hour post-stimulation, reporter activity was measured. (d) RAW 264.7 cells were transfected with both IL-10 reporter and PPAR $\gamma$ expression constructs. Twenty-four hour post-transfection, cells were stimulated as indicated and reporter activity was measured. (e) I/10 and Tnf mRNA expression in lung MDSCs isolated from LPS-treated mice and transfected ( $1 \times 10^{6}$ cells per condition) with scrambled or pparg-targeted siRNA (10 nM) was studied by qPCR (left and middle panel). IL-10 protein in culture supernatant was measured by ELISA (right panel). (f) Reporter assay of peritoneal macrophages transfected with plasmid constructs containing WT or deletion mutants of the IL-10 promoter (WT and mutants with deletions of indicated PPAR/RXR sites) linked to the luciferase gene. Statistical significance was calculated using one-way ANOVA with Bonferroni's post hoc test for multiple pairwise comparisons. Data shown are mean \pm s.d. and all data are representative of two independent experiments. ${ }^{\star} P \leq 0.05,{ }^{\star \star} P \leq 0.01$ and ${ }^{\star \star \star} P \leq 0.001 ; \mathrm{NS}$, not significant.

selected NF- $\kappa$ B-regulated genes via transrepression ${ }^{21,29}$. Transrepression plays an important role in inhibition of expression of pro-inflammatory genes like TNF and IL-6. However, in our present study, we observed a significant increase in TNF level in whole lung homogenate of mice treated with LPS + cardiolipin with concomitant reduction in IL-10 level (Fig. 2c). These observations in aggregate prompted us to hypothesize that SUMOylation of PPAR $\gamma$, induced by cardiolipin and rosi occur at distinct amino acid residues in PPAR $\gamma$ and this differential SUMOylation contributes to contrasting physiological outcomes. We then examined the recruitment of HDAC3 to the TNF URR in lung MDSCs treated with either cardiolipin or rosi following NF- $\kappa \mathrm{B}$ activation by LPS. Data from ChIP analysis showed marked recruitment of HDAC3 on the NF- $\kappa \mathrm{B}$ response element at $-214 \mathrm{bp}$ in the TNF URR in the presence of rosi but not cardiolipin (Fig. 5b, left panel). This observation was supported by another ChIP assay where LPS-induced p65 recruitment on the same NF- $\kappa B$ RE of TNF-URR was significantly reduced by rosi treatment but not with cardiolipin (Fig. 5b, left panel). The quantitative analysis of both HDAC3 and p65 ChIP assays performed by qPCR revealed similar trend (Fig. 5b, right panels). Collectively, these data confirmed that cardiolipin and rosi differentially regulate TNF, both involving PPAR $\gamma$.

Our next task was to investigate whether differential HDAC3 recruitment by cardiolipin versus rosi is due to distinct
SUMOylation of PPAR $\gamma$. The present literature revealed two SUMOylation sites in PPAR $\gamma$-one at lysine 107 (K107) and the other at lysine $395(\mathrm{~K} 395)^{21,30-33}$. K395 SUMOylation in PPAR $\gamma$ is involved in transrepression ${ }^{21}$, while SUMOylation at $\mathrm{K} 107$ represses gene transcription directly in cis by binding to PPREs ${ }^{33-35}$. To investigate which site was utilized by cardiolipin for HDAC3 recruitment to the IL-10 URR, PPAR $\gamma$ expression constructs containing point mutation in the individual SUMOylation sites were introduced in the RAW264.7 macrophage cell line (which lacks endogenous PPAR $\gamma$ expression) and the cells were stimulated with LPS \pm cardiolipin or rosi. Immunoprecipitated PPAR $\gamma$ was analysed for SUMOylation and HDAC3 association. Cardiolipin treatment following LPS stimulation failed to induce PPAR $\gamma$ SUMOylation and HDAC3 association when the K107 site was mutated (Fig. 5c). However, SUMOylation and HDAC3 recruitment were unchanged when the K395 SUMOylation site was mutated. In contrast, SUMOylation and HDAC3 recruitment were evident with rosi when the K107 site was mutated, but was lost when the K395 position was altered to arginine (Fig. $5 \mathrm{c}$ ). Figure $5 \mathrm{~d}$ shows no effect of the K395R mutation on cardiolipin-mediated inhibition of IL-10 production from the macrophages although the inhibition was lost when the K107R mutant was used. The results showed that SUMOylation of $\operatorname{PPAR} \gamma$ induced by cardiolipin and rosi involves different amino acid residues in 
a

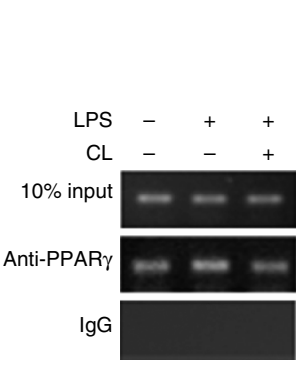

b

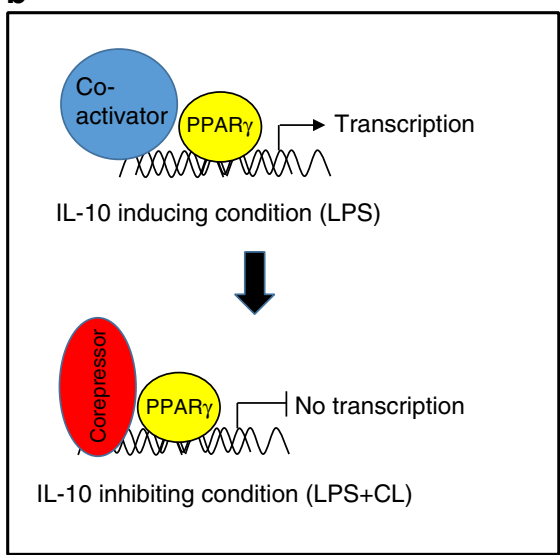

C

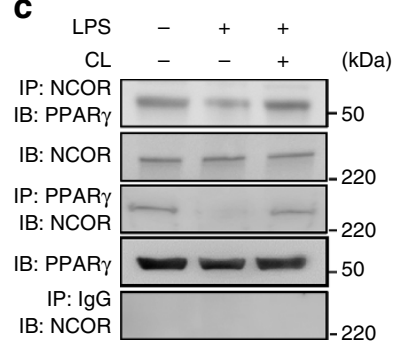

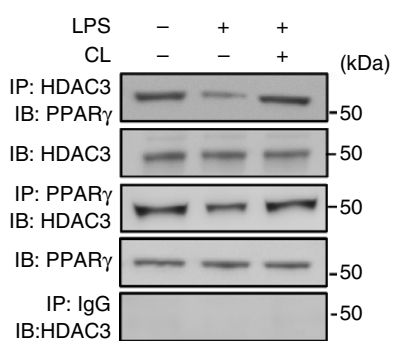

d

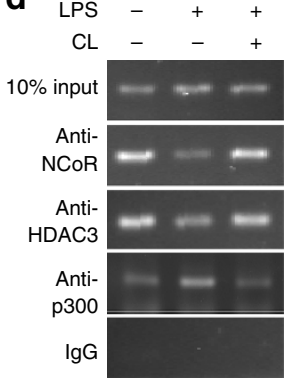

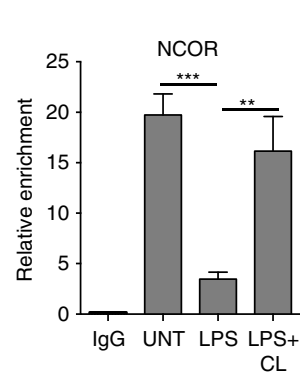

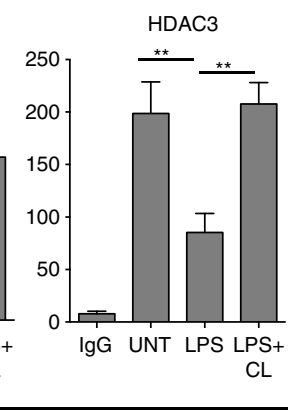

PPRE in IL-10 upstream

regulatory region

Figure 4 | Cardiolipin promotes association of corepressor complex with PPAR $\gamma$. Lung MDSCs were treated ex vivo with LPS (1 $\mu$ g ml ${ }^{-1}$ ) \pm cardiolipin $\left(10 \mu \mathrm{g} \mathrm{ml}^{-1}\right)$ for $2 \mathrm{~h}$ and binding of PPAR $\gamma$ to the IL-10 promoter and association with corepressor proteins were studied. (a) ChIP assay was performed using PPAR $\gamma$-specific antibody. Immunoprecipitated chromatin was used as template to amplify the region containing the PPRE at - 339 bp in the IL-10 URR (left panel). qPCR quantification of three independent ChIP experiments is shown (right panel). (b) Schematic of hypothesis of IL-10 regulation in lung MDSCs by LPS \pm cardiolipin. (c) NCOR- or HDAC3-associated protein complexes were immunoprecipitated using their respective antibodies and probed with PPAR $\gamma$-specific antibody or vice versa. (d) ChIP assay using NCOR-, HDAC3- or p300-specific antibody (left panel). qPCR quantitation of three independent ChIP experiments is shown (right panel). Statistical significance was calculated using one-way ANOVA with Bonferroni's post hoc test for multiple pairwise comparisons. Data shown are mean \pm s.d. and all data are representative of two independent experiments. ${ }^{\star} P \leq 0.05,{ }^{\star} P \leq 0.01$, ${ }^{\star \star \star} P \leq 0.001$ and ${ }^{\star \star \star \star} P \leq 0.0001 ;$ NS, not significant.

$\operatorname{PPAR} \gamma$ and provide a fundamental mechanism for differential TNF regulation by these two agents.

Cardiolipin metabolite cPA induces SUMOylation of PPAR $\gamma$. To understand how extracellular cardiolipin works on PPAR $\gamma$ we analysed the intracellular metabolites of cardiolipin in lung MDSCs. Isolated lung MDSCs were treated with ${ }^{3} \mathrm{H}$-labelled cardiolipin for different lengths of time and intracellular metabolites were analysed by thin layer chromatography. As shown in Fig. 6a, exposure of the cells to extracellular cardiolipin led to an increase in the levels of different lipid moieties including cPA. In view of the fact that $\mathrm{cPA}$ is a potent antagonist of PPAR $\gamma$ (ref. 36) we investigated whether like cardiolipin, cPA has the ability to induce SUMOylation of PPAR $\gamma$ with concomitant recruitment of HDAC3 to the IL-10 URR. Towards this end, we exposed lung MDSCs to purified cPA and examined SUMOylation of PPAR $\gamma$ by immunoprecipitation. A marked increase in SUMOylation of PPAR $\gamma$ was noticed in the cells treated with $\mathrm{CPA}$ which mimicked that induced by cardiolipin (Fig. 6b). Next we investigated whether cPA-mediated SUMOylation of PPAR $\gamma$ also led to the recruitment of HDAC3 to the IL-10 URR. ChIP analysis of lung MDSCs treated with $\mathrm{CPA}$ revealed a similar level of recruitment of HDAC3 to the IL-10 URR as induced by cardiolipin (Fig. 6c, left panel). Quantitation of ChIP experiments performed by qPCR using samples from two independent experiments is shown (Fig. 6c, right panel). Next we investigated the effect of cPA-induced recruitment of corepressors HDAC3 and NCOR on IL-10 expression, in lung MDSC cells. Similar to cardiolipin treatment, a marked suppression in the level of IL-10 was observed in the culture supernatant of cells treated with LPS and cPA (Fig. 6d). Our next goal was to investigate whether both cPA and cardiolipin use the same K107 amino acid residue of PPAR $\gamma$ for SUMOylation. With this objective, we transfected RAW264.7 macrophage cell line with either wild type or mutated PPAR $\gamma$ expression vector in which the K107 residue was mutated to arginine (K107R). Cells were stimulated with or without LPS \pm CPA, PPAR $\gamma$ was immunoprecipitated and SUMOylation was studied by western blotting methods. As shown in Fig. 6e, the marked induction of PPAR $\gamma$ SUMOylation by $\mathrm{cPA}$ treatment was reduced when cells were transfected with the mutant (K107R) PPAR $\gamma$ expression vector demonstrating that both cardiolipin and $\mathrm{CPA}$ use the same amino acid residue for PPAR $\gamma$ SUMOylation (Figs $5 \mathrm{c}$ and 6e). As shown in Supplementary Fig. 5, the lung MDSCs express the enzymes autotaxin and PLD2 that may be involved in the metabolism of cardiolipin to cPA. Collectively, these data suggest the involvement of cPA as a cardiolipin metabolite that mediates its biological effect of inhibition of IL-10 expression in MDSCs. 
a
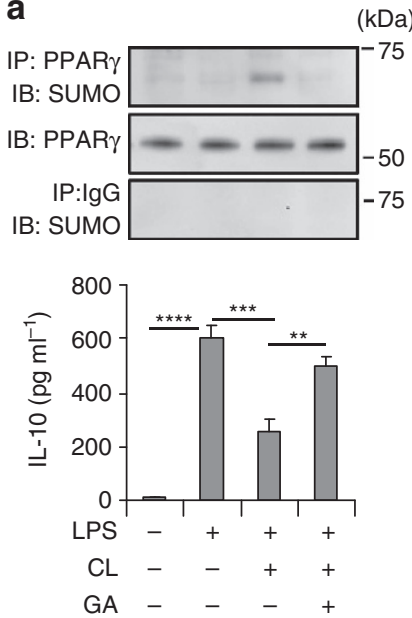

C

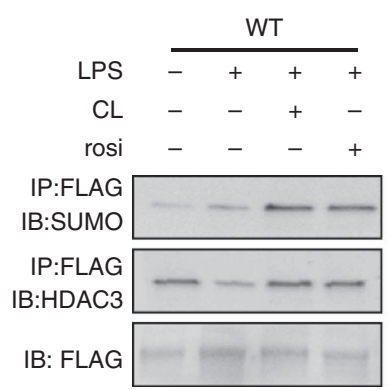

b

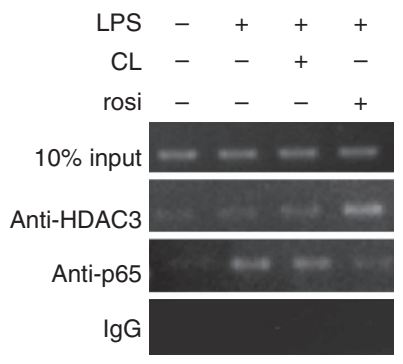

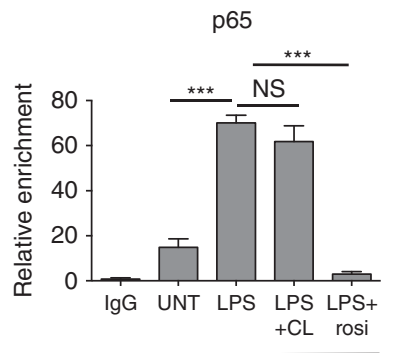

NF-KB RE in TNF upstream regulatory region
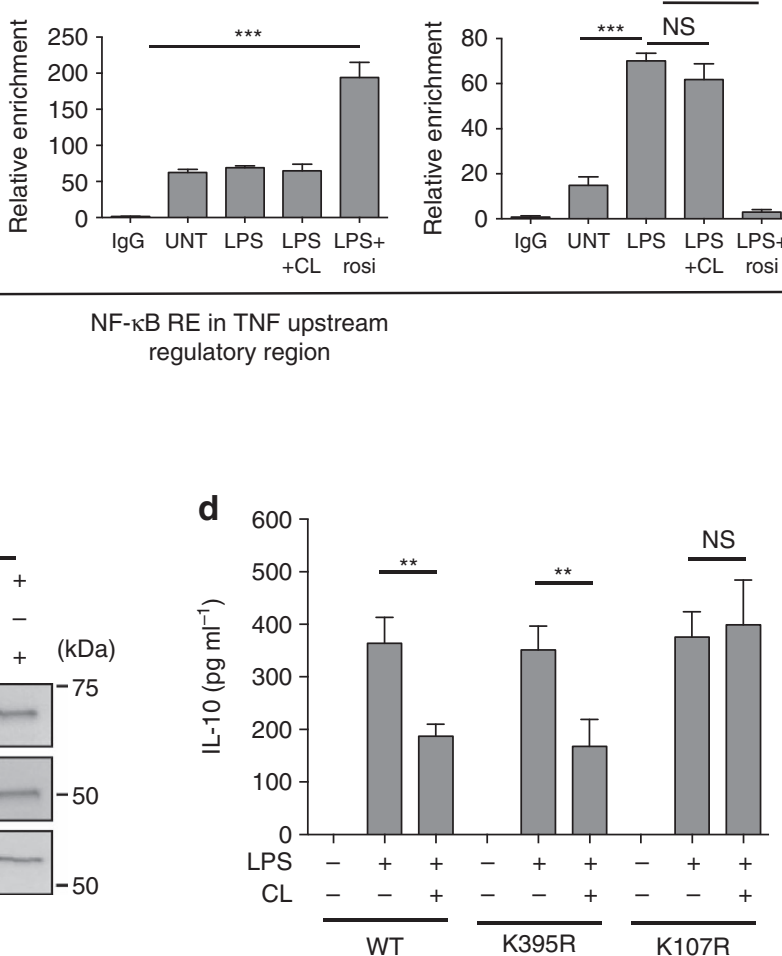

Figure 5 | Cardiolipin-induced PPAR $\boldsymbol{\gamma}$-SUMOylation is required to recruit corepressors to the IL-10 URR. (a) Lung MDSCs were treated individually or in combination with LPS, cardiolipin or ginkgolic acid (GA, 50 $\mu \mathrm{M})$ for $6 \mathrm{~h}$. SUMOylation of immunoprecipitated PPAR $\gamma$ was studied by western blot analysis (upper panel) and IL-10 in the culture supernatant was measured by ELISA (lower panel). (b) Lung MDSCs were treated with LPS, cardiolipin or rosiglitazone (rosi) either alone or with the indicated combinations. ChIP assay was performed with antibodies against either HDAC3 or the p65 subunit of NF- $\kappa B$ (RelA). The region containing the NF- $\mathrm{kB}$ response element at $-214 \mathrm{bp}$ in the TNF promoter was amplified with specific primers. qPCR quantitation represents analysis of two independent ChIP experiments (middle and right panels) (c,d) RAW macrophages were transfected with plasmid expression constructs containing either FLAG-tagged WT PPAR $\gamma$ gene (left panel) or a variant containing either K395R (middle panel) or K107R (right panel) mutation in PPAR $\gamma$. Transfected cells were treated individually or in combination with LPS, cardiolipin or rosi. FLAG-tagged PPAR $\gamma$ was immunoprecipitated with anti-FLAG antibody. SUMOylation and HDAC3 association with PPAR $\gamma$ was studied by western blot analysis. (d) Secreted IL-10 in the culture supernatant was measured by ELISA. Statistical significance was calculated using one-way ANOVA with Bonferroni's post hoc test for multiple pairwise comparisons. Data shown are mean \pm s.d. and all data are representative of two independent experiments. ${ }^{\star \star} P \leq 0.01$ and ${ }^{\star \star \star} P \leq 0.001$; NS, not significant.

HDAC inhibition restores IL-10 with resolution of pneumonia. Because our data suggested that the recruitment of HDAC3 to the IL-10 URR is critical for cardiolipin-mediated IL-10 suppression, we investigated whether inhibition of HDAC activity in vivo using the short-chain fatty acid, sodium butyrate $(\mathrm{NaB})^{37}$, could overcome IL-10 suppression. To this end, we treated mice with $\mathrm{NaB}$ for five consecutive days, initiated a day after treatment with LPS + cardiolipin. Among the LPS + cardiolipin-treated mice, the group of mice that received $\mathrm{NaB}$ showed significant gain in body weight (Fig. 7a), restoration of normal lung histology (Fig. 7b), reduced MPO activity in BAL fluid (Fig. 7c), significant reduction in lung leakage (Fig. 7d) and increased IL-10 but decreased TNF levels in the lung (Fig. 7e) as compared with the vehicle-treated group. ChIP analysis of lung MDSCs isolated from NaB-treated mice showed significantly greater recruitment of acetylated Histone 3 (acetyl H3) to the IL-10 URR (Fig. 7f, upper panel). Quantitation of ChIP experiments performed by qPCR yielded similar data (Fig. 7f, lower panel). This observation was in line with the increased level of IL-10 in the whole lung (Fig. 7e). Since $\mathrm{NaB}$ inhibits multiple HDACs including HDAC3, we were interested to determine the extent to which the therapeutic potential of $\mathrm{NaB}$ was dependent on its role in rescuing IL-10 suppression. To this end we treated $1 l 10^{-/-}$ mice with $\mathrm{NaB}$ for five consecutive days, initiated a day after treatment with LPS + cardiolipin. In NaB-treated $I l 10^{-1-}$ mice, we did not observe improvement of lung histology as compared with vehicle-treated $1 l 10^{-/-}$mice (Fig. $7 \mathrm{~g}$ ). Moreover, both groups of mice showed comparable BAL MPO activity (Fig. 7h) and lung leakage (Fig. 7i). These data suggest that although $\mathrm{NaB}$ inhibits multiple HDACs including HDAC3, in our experimental model the therapeutic potential of $\mathrm{NaB}$ is primarily due to its role in rescuing IL-10 suppression by inhibiting HDAC3.

With our ultimate goal being to understand the mechanism of compromised IL-10 expression under conditions of non-resolving bacterial pneumonia, we verified involvement of this mechanism in mice infected with $K$. pneumoniae. IP performed with lung MDSCs isolated from mice infected with high dose (1,000 c.f.u.) of Klebsiella revealed increased SUMOylation of $\operatorname{PPAR} \gamma$ which was not evident in mice treated with a low dose (100 c.f.u.) of bacteria (Fig. 8a). Increased SUMOylation was coupled with augmented association of PPAR $\gamma$ with HDAC3 in the same group of mice (Fig. 8a). Also, when mice were infected 
a $\quad 1 \quad 2 \quad 3 \quad 3 \quad 4$

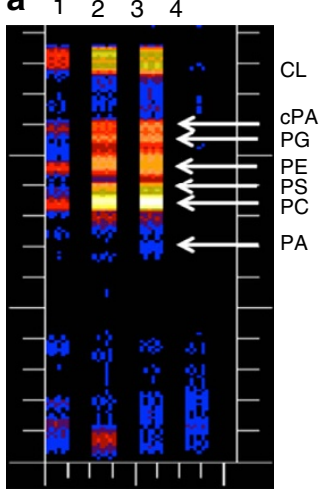

b

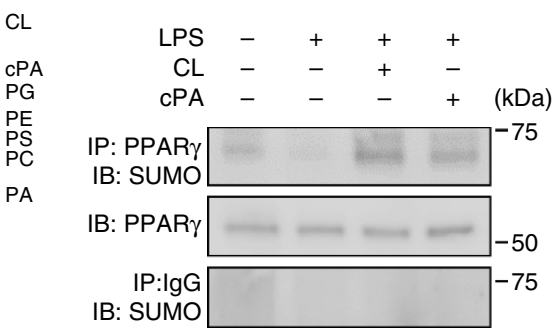

C

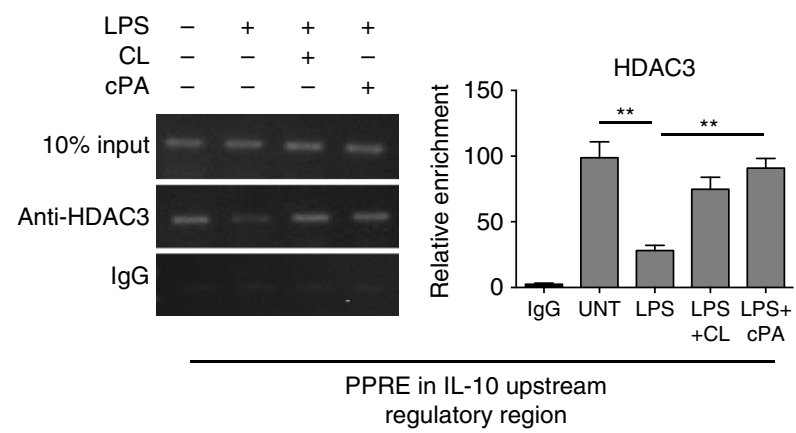

e

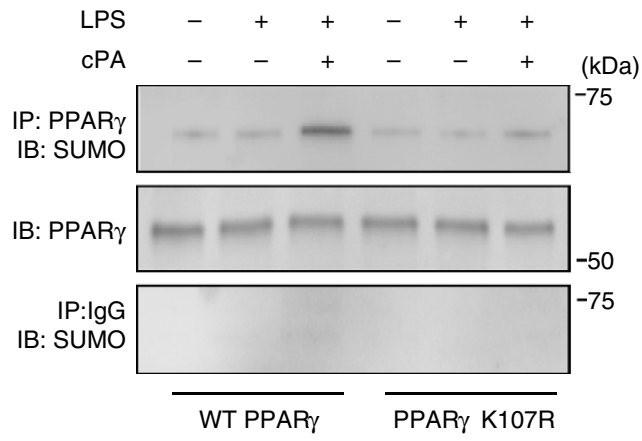

Figure 6 | The cardiolipin metabolite CPA induces SUMOylation of PPAR $\gamma$ and recruitment of corepressor complex. (a) Lung MDSCs were incubated with ${ }^{3} \mathrm{H}$-labelled cardiolipin for 10, 30 and $60 \mathrm{~min}$ and cellular lipids were extracted using the Bligh and Dyer method. Lipids were then resolved by thin layer chromatography to detect individual phospholipids. The ${ }^{3} \mathrm{H}$ metabolites of cardiolipin were quantified using a plate reader. Lanes 1, 2 and 3 depict incubation of ${ }^{3} \mathrm{H}$-labelled cardiolipin for 10, 30 and 60 min, respectively, lane 4-blank. (b-d) Lung MDSCs were treated with either LPS (1 $\mu \mathrm{g} \mathrm{ml}{ }^{-1}$ ) alone or in combination with cardiolipin $\left(10 \mu \mathrm{g} \mathrm{ml}^{-1}\right)$ or CPA $(50 \mu \mathrm{M})$ and analysed. (b) PPAR $\gamma$ was immunoprecipitated and SUMOylation was studied by western blot methods. (c) HDAC3 recruitment to the IL-10 URR was investigated by ChIP assay (left panel). qPCR quantitation represents analysis of three independent ChIP experiments (right panel). (d) IL-10 in cell-free culture supernatant was measured by ELISA. (e) RAW 264.7 cells were transfected with either wild type or mutated (K107R) PPAR $\gamma$ (FLAG-tagged) expression construct. Twenty-four hour post-transfection, cells were stimulated with LPS \pm cPA as indicated. Two hours post-stimulation, PPAR $\gamma$ was immunoprecipitated and SUMOylation was examined by western blot methods. Statistical significance was calculated using one-way ANOVA with Bonferroni's post hoc test for multiple pairwise comparisons. Data shown in all experiments are mean \pm s.d. and data are representative of two independent experiments. ${ }^{\star \star} P \leq 0.01$; NS, not significant.

with the low dose of bacteria (100 c.f.u.), HDAC3 and NCOR recruitment to the IL-10 URR was lower compared with recruitment of the coactivator, p300 (Fig. 8b). However, infection with a higher dose of bacteria (1,000 c.f.u.) yielded opposite data with increased recruitment of NCOR/HDAC3 and decreased recruitment of p300 (Fig. 8b). Quantitation of ChIP assays for HDAC3, NCOR and p300 was performed by qPCR using samples from two independent experiments and the results are shown below Fig. 8b. Similarly, high but not low dose of bacteria suppressed IL-10 levels in the lung (Fig. 8c). We next examined the effect of $\mathrm{NaB}$ in mice infected with $K$. pneumonia (1,000 c.f.u.). Similar to data obtained in mice treated with LPS + cardiolipin, NaB significantly inhibited loss in body weight, caused increased survival, inhibited lung pathology and significantly reduced lung leakage, the combination was the most effective (Fig. $8 \mathrm{~d}-\mathrm{g}$ ). $\mathrm{NaB}$ treatment also led to an increase in lung IL-10 level and a decrease in TNF level (Fig. 8h). Taken together, these data reveal a dynamic interplay between PPAR $\gamma$ SUMOylation and corepressor/HDAC and coactivator to regulate IL-10 production, which is compromised by cardiolipin and the ability of $\mathrm{NaB}$ to overcome the cardiolipin-induced adverse effects.

\section{Discussion}

Non-resolving lung inflammation induced by bacterial infection is still a leading cause of morbidity and mortality worldwide.
Continuous stimulation through TLRs, increased cell death and poor clearance of apoptotic neutrophils are considered to be the major contributors to aggravated lung inflammation ${ }^{1}$. However, it is not well understood why anti-inflammatory responses fail under conditions of non-resolving pneumonia. Here we show that the phospholipid cardiolipin, previously shown to be elevated in the lungs of humans with bacterial pneumonia $^{17}$, and also in the lungs of mice infected by bacteria (present study), blunts IL-10 production from lung MDSCs and perpetuates lung inflammation. We expect that non-resolving lung inflammation associated with other Gram-negative bacteria would involve similar mechanisms of corepressor recruitment to the IL-10 promoter since our observations were essentially similar with LPS + cardiolipin and $K$. pneumoniae. The immunomodulatory role of MDSCs in cancer is well recognized where they suppress anti-tumour immune responses and promote tumour progression and metastasis ${ }^{38,39}$. However, a beneficial role of MDSCs in terminating infection-associated inflammation is less studied but being increasingly appreciated ${ }^{23}$. We show an important role of PPAR $\gamma$ in promoting IL-10 gene expression in the MDSCs and the ability of cardiolipin to impair this process by inducing SUMOylation of PPAR $\gamma$ causing recruitment of an NCOR/HDAC corepressor complex to the IL-10 promoter without affecting the TNF promoter. The PPAR $\gamma$ SUMOylation at K107 identified in our study is distinct from the well-described K395 SUMOylation that can be induced by 

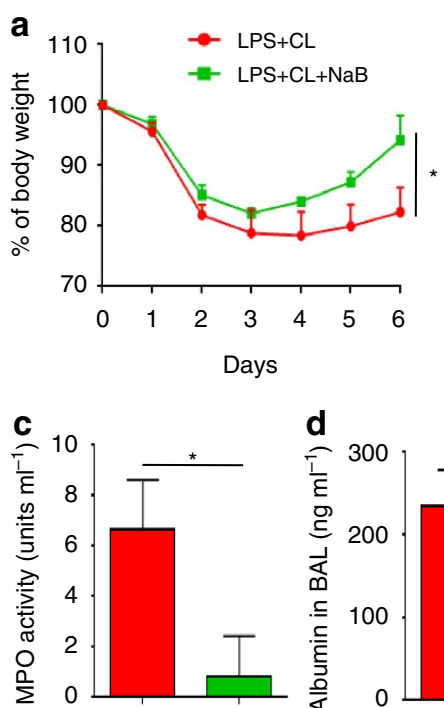

Day 6

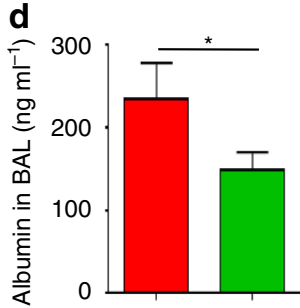

Day 6

b
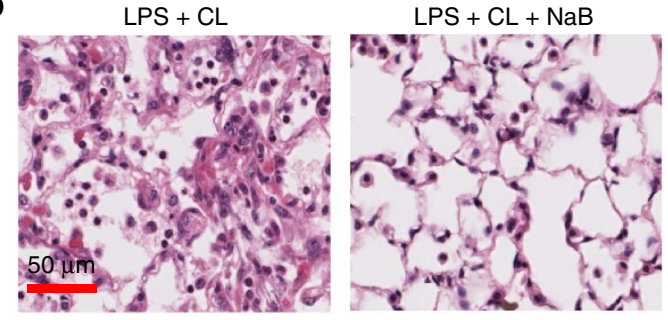

e

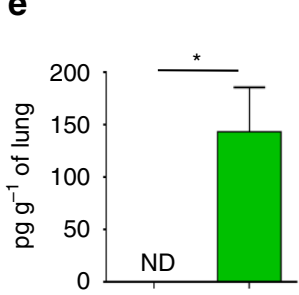

IL-10

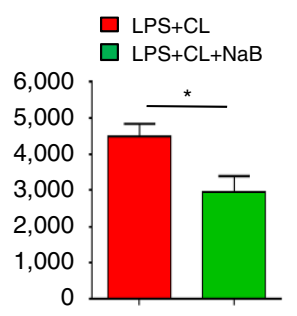

TNF g

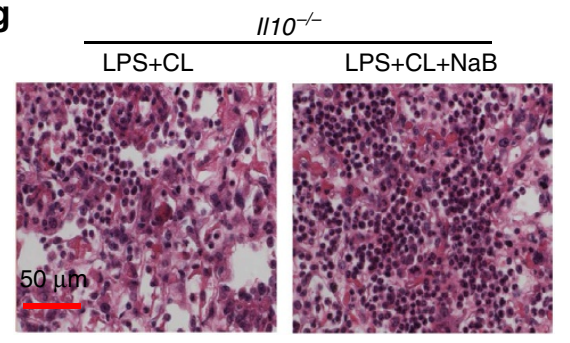

h

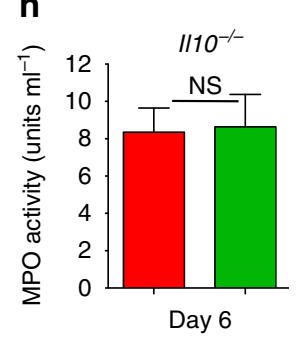

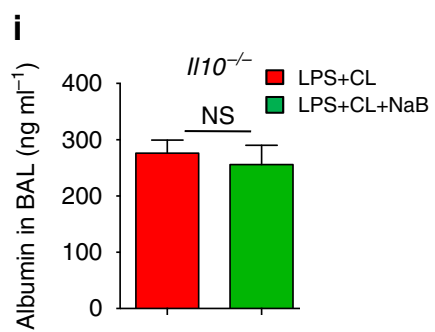

Day 6

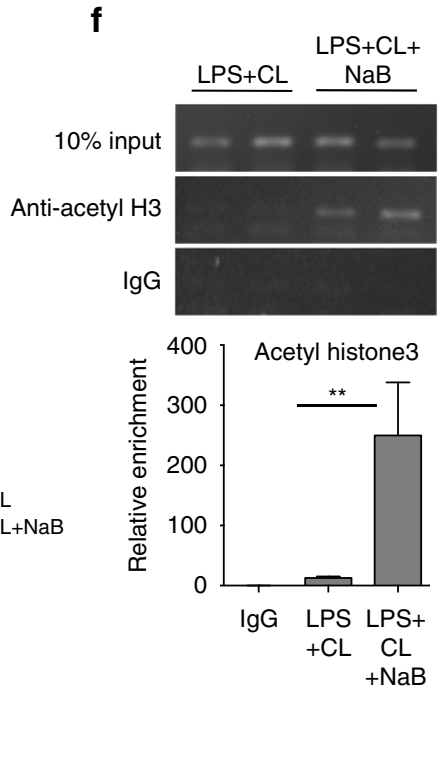

Figure 7 | Inhibition of HDAC3 by a short-chain fatty acid promotes resolution of lung inflammation. C57BL/6J mice were treated i.t with both LPS (20 $\mu \mathrm{g}$ per mouse) and cardiolipin (100 $\mu \mathrm{g}$ per mouse). Day 1 post-treatment, mice were randomly divided into two groups. One group was treated i.p. with an HDAC3 inhibitor, sodium butyrate $(\mathrm{NaB})\left(200 \mathrm{mg} \mathrm{kg}^{-1}\right.$ body weight) for five consecutive days while the other group received only vehicle (PBS). Day 6 post-treatment, the following parameters of inflammation were studied: (a) Change in body weight, (b) lung pathology by H\&E staining of lung tissue sections, (c) MPO activity in BAL fluid, (d) BAL albumin level and (e) Secreted cytokines (IL-10 and TNF) assayed in lung homogenates using magnetic bead-based assay. (f) Lung MDSCs were isolated from the two groups of mice and ChIP assay was performed to study recruitment of acetylhistone to the IL-10 URR (upper panel). qPCR quantitation represents analysis of two independent $\mathrm{ChIP}$ experiments (lower panel). ( $\mathbf{g}^{-\mathbf{i}}$ ) I/10-/- mice were treated i.t. with both LPS and cardiolipin as described above. Day 1 post-treatment, mice were randomly divided into two groups. One group was treated (i.p.) with an HDAC3 inhibitor, sodium butyrate $(\mathrm{NaB})$, while the other group received only vehicle (PBS). Day 6 post-treatment, the following parameters of inflammation were studied: ( $\mathbf{g}$ ) lung pathology by H\&E staining of lung tissue sections, (h) MPO activity in BAL fluid, (i) BAL albumin level by ELISA. Statistical significance was calculated using Student's unpaired two-tailed $t$ test for comparisons involving two groups or one-way ANOVA with Bonferroni's post hoc test for multiple pairwise comparisons. The experiment was initiated with 8 mice in LPS + cardiolipin group and 6 mice in LPS + cardiolipin + NaB group with 3 and 4 mice respectively in each group available for analysis at the conclusion of the experiment. Data shown are mean \pm s.d. and are representative of two independent experiments. ${ }^{\star} P \leq 0.05,{ }^{\star}{ }^{\star} P \leq 0.01$; NS, not significant.

PPAR $\gamma$ agonists such as the thiazolidinedione, rosi. Our data show that cardiolipin differentially influences expression of anti- (IL-10) versus pro- (TNF)-inflammatory cytokines via selective recruitment of corepressor complex to the IL-10 promoter region. We show beneficial effect of HDAC inhibition using a short-chain fatty acid, $\mathrm{NaB}$, in the lungs of infected mice, which restored IL-10 levels but suppressed TNF levels with accompanying gain in body weight and survival of the animals.

In our efforts to determine how a high level of cardiolipin generated in the lungs of mice infected by bacteria inhibits IL-10 production, we detected accumulation of intracellular metabolites like cPA when cells were exposed to cardiolipin, which highlights a mechanism by which extracellular cardiolipin can exert deleterious effects on important immunoregulatory mechanisms. This is because cPA was previously shown to inhibit PPAR $\gamma$ activation by rosi due to competitive binding of $\mathrm{cPA}$ to the active site of PPAR $\gamma$ (ref. 36). We show similar inhibitory effects of cardiolipin and cPA on IL-10 production from lung
MDSCs, both being lost when K107 in PPAR $\gamma$ is mutated, this residue being a known SUMOylation target and is involved in cis-activating functions of PPAR $\gamma$ (refs 33-35). Although not the goal of the present study, since cardiolipin can be metabolized to cPA, we do expect cardiolipin to function as an antagonist of rosi-activated PPAR $\gamma$ as previously reported ${ }^{36}$. It is interesting to note similar end results of targeting cPA in the two studies; while blocking cPA generation using PLD2 shRNA relieved cPA-mediated suppression of the PPAR $\gamma$ target genes fabp 4 and $c d 36$ in the previous study ${ }^{36}$, in our experiments, blocking cardiolipin/cPA function by blocking K107 SUMOylation using ginkgolic acid relieved suppression of IL-10 production (Fig. 5a).

The key finding in our study is the ability of cardiolipin to inhibit anti-inflammatory mechanisms such as IL-10 production from lung MDSCs, which is the principal source of IL-10 during bacterial infection. Compromised IL-10 production observed in the presence of cardiolipin was associated with higher numbers of infiltrating cells, which persisted in the lung. Thus, 
a

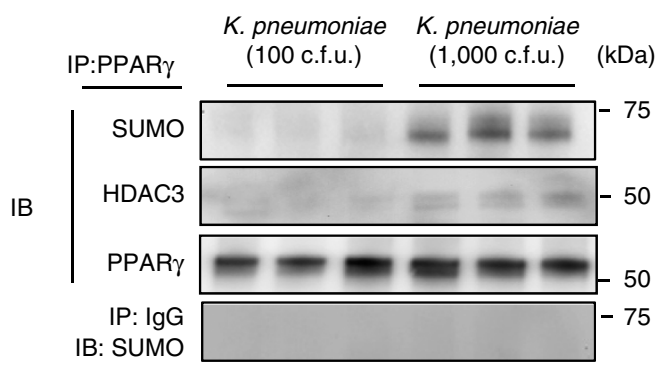

b

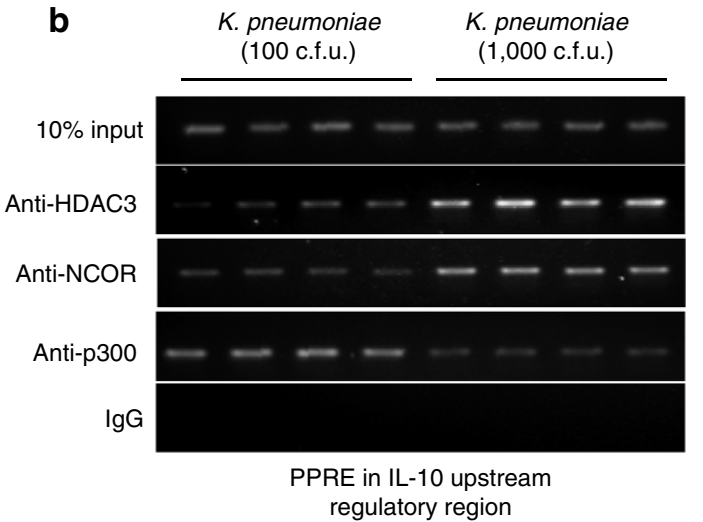

C
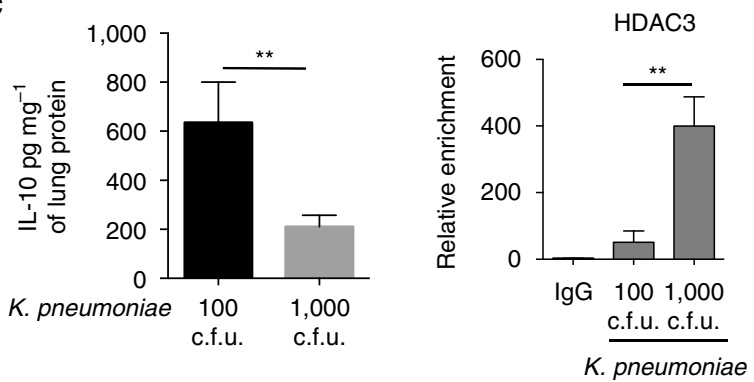
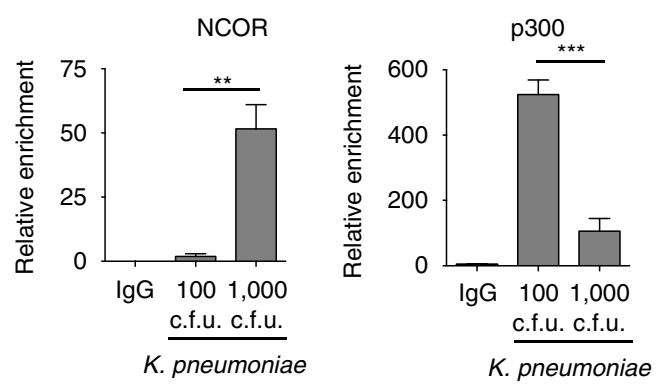

d
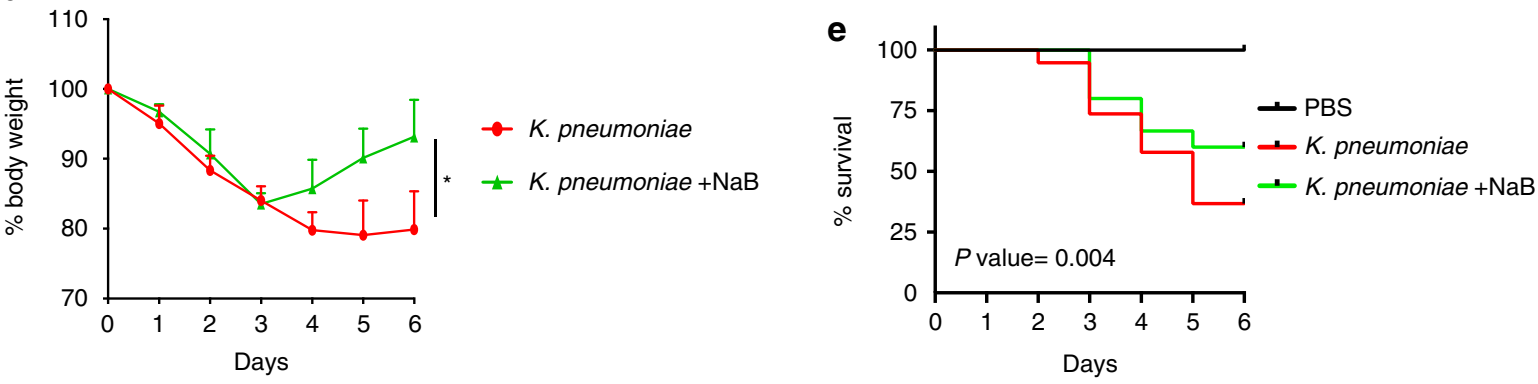

f

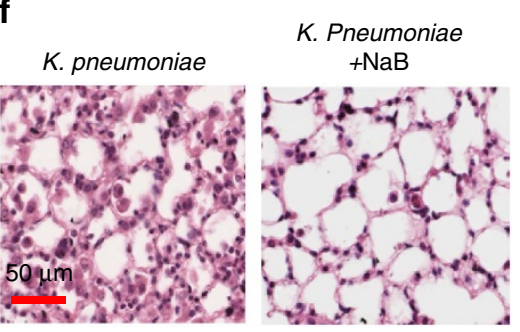

g

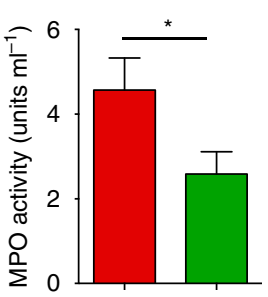

h

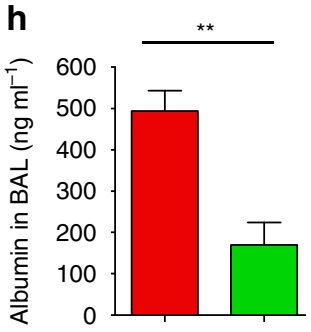

K. pneumoniae

i

Kneumoniae $+\mathrm{NaB}$

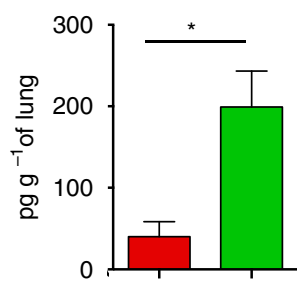

IL-10

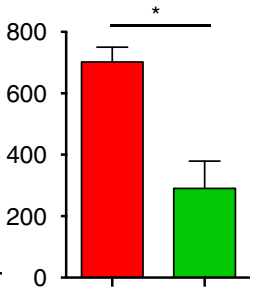

TNF

Figure 8 | Infection with high dose of $K$. pneumoniae recruits corepressor complex to the IL-10 URR and amelioration of pathogenicity by short-chain fatty acid. (a-c) C57BL/6J mice were infected i.t. with either low dose (100 c.f.u.) or high dose (1,000 c.f.u.) of K. pneumoniae ( $n=3-4$ mice per group at the conclusion of experiment). Four days post-infection, lung MDSCs were purified. (a) Coimmunoprecipitation and (b) ChIP experiments were performed using NCOR-, HDAC3- or p300-specific antibodies. Data shown are representative of two independent experiments. qPCR quantitation represents analysis of two independent ChIP experiments (lower panel). (c) IL-10 protein level in the lung on day 6 was measured by magnetic bead-based cytokine assay. Data shown are mean \pm s.d. and representative of two independent experiments. (d-i) C57BL/6J mice were infected with 1,000 c.f.u. of K. pneumonia. Day 1 post-infection, mice were randomly divided into two groups. One group was treated i.p. with $\mathrm{NaB}$ ( $200 \mathrm{mg} \mathrm{kg}^{-1}$ body weight) for five consecutive days while the other group received only vehicle (PBS). Day 6 post-treatment, the following parameters of inflammation were studied: (d) Change in body weight. (e) Percentage of survival represented by Kaplan-Meier survival curves of PBS-treated mice or mice infected with Kleb \pm NaB treatment. Data shown are mean \pm s.d. from three pooled experiments with $n=12$ mice in PBS group, 15 in bacteria only group and 20 in bacteria + NaB group. The $P$ value was calculated using log-rank (Mantel-Cox) test. (f) Lung pathology by H\&E staining of lung tissue sections. (g) MPO activity in BAL fluid, (h) BAL albumin level by ELISA, and (i) secreted cytokines assayed in lung homogenates using magnetic bead-based assay. Experiments in $\mathbf{c}, \mathbf{d}$ and $\mathbf{f}-\mathbf{i}$ were initiated with $n=8$ and 6 mice in Klebsiella and Klebsiella + NaB groups, respectively. At the conclusion of the experiment, 3 mice in bacteria only group and 4 mice in bacteria $+\mathrm{NaB}$ group were available for analysis. Statistical significance was calculated using Student's unpaired two-tailed $t$ test for comparisons involving two groups or one-way ANOVA with Bonferroni's post hoc test for multiple pairwise comparisons. Data shown are mean $\pm \mathrm{s}$.d. and are representative of two independent experiments. ${ }^{\star} P \leq 0.05,{ }^{\star \star} P \leq 0.01,{ }^{\star \star \star} P \leq 0.001$. 
cardiolipin can impair resolution of inflammation by antagonizing a key anti-inflammatory immune response during bacterial infection ${ }^{16}$. Cardiolipin has been shown to directly exert pro-inflammatory effects by promoting NLRP3 inflammasome activation $^{40}$ and in this context too IL-10 serves an important anti-inflammatory function by inhibiting NLRP3-mediated inflammasome activation ${ }^{41,42}$.

Although PPAR $\gamma$ activation by a synthetic ligand ${ }^{27}$ or a gut bacterium ${ }^{28}$ was previously associated with increased IL-10 production in the gut mucosa, our study provides direct molecular proof of PPAR $\gamma$ utilization during IL-10 gene expression. Since IL-10 plays a central role in resolution of inflammation, its regulation has been studied in several immune cell types including $\mathrm{T}$ cells, dendritic cells and macrophages ${ }^{43}$. Multiple transcription factors including SP1 (ref. 44), MAF $^{43,45,46}$, GATA-3 (refs 47,48), ATF2, CREB ${ }^{49}$ and $N F-\kappa B^{50,51}$ have been implicated in IL-10 gene expression, which collectively illustrates cell-specific mechanisms of IL-10 gene transcription. IL-10 regulation in lung MDSCs, however, is not well understood. In the present study, we have identified a novel mechanism of IL-10 gene expression involving PPAR $\gamma$ that does not involve the well-studied transrepression function of PPAR $\gamma$ and yet contributes to the anti-inflammatory actions of PPAR $\gamma$ by promoting IL-10 production. Of note, while our study demonstrates an essential role of PPAR $\gamma$ in mediating IL-10 gene expression in the lung MDSCs, it is likely that PPAR $\gamma$ acts in concert with other transcription factors to induce optimal transcription of IL-10. We have observed that under basal conditions, PPAR $\gamma$ is constitutively bound to the IL-10 upstream regulatory region along with the corepressors HDAC3 and NCOR. Interaction of members of the nuclear receptor family with corepressor molecules under basal conditions has been reported for the thyroid hormone receptor and the retinoid $\mathrm{X}$ receptor ${ }^{52-55}$ but has not been demonstrated for $\operatorname{PPAR} \gamma$ heretofore. This mechanism plays a critical role in restricting basal IL-10 expression, which has physiological significance. Our study and those of others have shown that during bacterial infection, early expression of IL-10 is detrimental since it inhibits the recruitment of leukocytes, which is necessary for bacterial clearance $8,16,56$. However, delayed expression of IL-10 is equally important to terminate the ongoing inflammatory response to prevent tissue injury ${ }^{16}$.

Anti-inflammatory function of PPAR $\gamma$ has been studied in multiple inflammatory diseases including rheumatoid arthritis, diabetes, inflammatory bowel disease and atherosclerosis in which PPAR $\gamma$ agonists such as rosi have been used $^{57-59}$. In these studies, transrepression of NF- $\kappa B$-regulated genes by $\operatorname{PPAR} \gamma$ was shown to be the key anti-inflammatory mechanism. As previously shown, rosi-mediated SUMOylation at K395 of PPAR $\gamma$ is important for transrepression functions of $\operatorname{PPAR} \gamma$ that involves recruitment of corepressor-associated $\operatorname{PPAR} \gamma$ to NF- $\kappa \mathrm{B}$ binding sites through protein-protein interactions thereby blocking transactivation functions of NF- $\kappa \mathrm{B}$ (refs 21,29). Major pro-inflammatory genes that are regulated by transrepression include TNF, iNOS and IL-6. However, in our present study we did not observe any suppression of TNF and IL-6 with cardiolipin treatmentinstead, the levels of both cytokines significantly increased in cardiolipin + LPS-treated mice as compared with that in mice treated with LPS alone. These results showed that SUMOylation of PPAR $\gamma$ induced by cardiolipin and that induced by rosi are distinct. Here, it is important to note that basal regulation of IL-10 does not require PPAR $\gamma$ SUMOylation while it is absolutely necessary to restrict its expression in the presence of cardiolipin. Cardiolipin promotes enrichment of corepressor/HDAC complex over that of a co-activator on the IL-10 promoter but spares the
TNF promoter, the net effect being persistent inflammation. Emerging literature suggests an important role of posttranslational modifications of PPAR $\gamma$ in regulating PPAR $\gamma$ activity impacting multiple disease conditions $31,57,60$ However, a role for PPAR $\gamma$ post-translational modification in promoting non-resolving lung inflammation by altering the coactivator:corepressor balance at the regulatory region of an important immunosuppressive gene has not been previously described.

Health benefits of short-chain fatty acids (SCFAs) are being increasingly appreciated where the major focus is improvement of gut-health $^{61,62}$. Here we show that use of the SCFA NaB is highly effective in the context of persistent lung inflammation during bacterial pneumonia. A protective effect of $\mathrm{NaB}$ was also observed in severe burn-induced lung injury in rats ${ }^{63}$. Our study highlights a new opportunity for future drug development using SCFAs as an adjunct therapy to ameliorate non-resolving pneumonia by maintaining IL-10 expression and reducing expression of pro-inflammatory cytokines like TNF.

\section{Methods}

Mice. To address our objective of how cardiolipin impairs resolution of lung inflammation we set up experimental mouse groups based on our original pilot experiments of bacterial infection using an LD50 dose in which assuming a two-sided $\alpha$ of 0.05 , four animals in each group are required to detect a $15-20 \%$ reduction in body weight on day 6 after infection with $80 \%$ power. We also used a model of non-resolving lung inflammation using a low dose of LPS in combination with cardiolipin. Having used a bacterial LD50 dose for infection after day 6 with similar inflammation induced by LPS + cardiolipin, we always initiated our experiments with eight animals in each group to accommodate attrition during the course of the study. We also conducted experiments to study the effect of the HDAC inhibitor, $\mathrm{NaB}$, on lung inflammation and loss in body weight of the animals after infection or treatment with LPS + cardiolipin. Mice were randomized for treatment. However, the experimenter was not blinded to the identity of the groups. Since we expected 50\% loss of animals due to use of LD50 dose of infection, animals that fit criteria for euthanasia after day 3 of infection, were killed in compliance with a protocol approved by our Institutional Animal Care and Use Committee at the University of Pittsburgh.

Male C57BL/6 J mice, and $I l 10^{-1-}$ mice on C57BL/6 J background (Stock \# 002251), were purchased from The Jackson Laboratory (Bar Harbor, ME). All mice were housed under pathogen-free conditions and were used between 6 and 10 weeks of age. All studies with mice were approved by the Institutional Animal Care and Use Committee at the University of Pittsburgh.

Reagents. Rosiglitazone (Cat \# R2408), GW9662 (Cat \# M6191), ginkgolic acid (Cat \# 75741), sodium butyrate (Cat \# B5887) and LPS (from Escherichia coli, strain O26:B6) (Cat \# L8274), were obtained from Sigma. Cardiolipin was purchased from Avanti polar lipids, (Cat \# 840012C), the major cardiolipin species being $(18: 2)_{4}$ or tetralinoleyl cardiolipin. GM-CSF was purchased from Peprotech (Cat \# 300-03). Antibodies against HDAC3 (polyclonal, Cat \# ab7030),

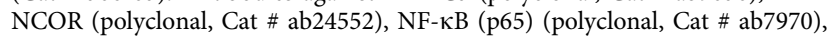
p300 (clone NM11, Cat \# ab3164), acetyl-Histone3 (polyclonal, Cat \# ab4441) and PPAR $\gamma$ (polyclonal, Cat \# ab19481) were purchased from Abcam and the anti-SUMO antibody (polyclonal, Cat \# 4930) was from Cell Signaling technology, USA. Antibodies were used at 1:1,000 dilution for western blotting and at 1:50 dilution for immunoprecipitation and ChIP assays. For flow cytometry, antibodies used were against IL-10 (clone JESS-16E3, either PE-conjugated, Cat \# 561060, from BD Biosciences used at 1:200 dilution or PercP-Cy5.5conjugated, Cat \# 505027 from Biolegends used at 1:200 dilution), CD11b (PerCP-Cy5.5-conjugated, clone M1/70, Cat \# 561114, used at 1:500 dilution), Ly6C (FITC-conjugated, clone AL-21, Cat \# 553104, at 1:500 dilution), and Ly6G (APC-conjugated, clone 1A8, Cat \# 560599) all purchased from BD Biosciences, and F4/80, (clone BM8, either AF488-conjugated clone BM8, Cat \# MF48020 from Biolegend or PE-conjugated, Cat \# MF48004 from Life Technologies both used at 1:400 dilution).

Cell isolation and sorting. To isolate lung MDSCs, mice were treated with LPS (10 $\mu \mathrm{g}$ per mouse) for 3 consecutive days. Twenty-four hours post-treatment, the lungs of anaesthetized mice were perfused with sterile PBS, removed and digested using methods previously described ${ }^{16}$. Briefly, lungs were dissociated in a collagenase-DNase suspension on a gentle MACS Dissociator (MiltenyiBiotec) according to the manufacturer's protocol. Single-cell suspensions were obtained by passing the dissociated tissue through a $70 \mu \mathrm{m}$ cell strainer (BD Falcon) and washed with PBS containing $2 \%$ FBS. CD11b ${ }^{+}$cells were enriched using

$\mathrm{CD} 11 \mathrm{~b}$ antibody-coated magnetic beads from Miltenyi Biotec. Enriched cells were 
sorted to $90-95 \%$ purity by flow cytometry using fluorochrome-labelled CD11b, F480 and Ly6G antibodies (BD Biosciences). Thioglycollate-elicited peritoneal macrophages were isolated day four post-treatment from mice injected intraperitoneally (i.p.) with $2 \%$ thioglycollate (Sigma, Cat \#T9032)

In vivo and ex vivo treatments. Male C57BL/6J mice were treated i.t. with either LPS ( $20 \mu \mathrm{g}$ per mouse), alone or in combination with cardiolipin (100 $\mu \mathrm{g}$ per mouse). Cardiolipin was administered $1 \mathrm{~h}$ post LPS instillation. Our experiments having shown cardiolipin levels in the lungs of infected mice in the range of $10-50 \mu \mathrm{g}$ per lung, we used a dose of $100 \mu \mathrm{g}$ of cardiolipin to maximize availability of cardiolipin in the lungs of all mice after i.t. instillation. Lung MDSCs were treated ex vivo with or without LPS $\left(1 \mu \mathrm{g} \mathrm{ml}^{-1}\right)$. One hour post LPS-treatment, cardiolipin $\left(10 \mu \mathrm{g} \mathrm{ml}^{-1}\right)$ was added to the culture medium. For ChIP, immunoprecipitation and western blot experiments, cells were harvested $2 \mathrm{~h}$ post cardiolipin treatment while for ELISA, culture supernatants were collected $6 \mathrm{~h}$ post cardiolipin stimulation.

LC/MS analysis of cardiolipin. Mice were either left uninfected or infected with 1,000 c.f.u. of $K$. pneumoniae. Day 3 post infection lipids were extracted from lung homogenates using the Folch protocol ${ }^{64}$ and phospholipid phosphorus was estimated by a micro-method ${ }^{65}$. LC/MS of cardiolipins was performed as previously described ${ }^{66}$. Briefly, LC/MS in negative mode was performed using a DionexUltimate 3,000 HPLC coupled on-line to a Q-Exactive hybrid quadrupoleorbitrap mass spectrometer (ThermoFisher Scientific, San Jose,

CA, USA). Total lipids were separated on a normal phase column (Silica Luna $3 \mu \mathrm{m}, 100 \AA, 150 \times 2 \mathrm{~mm}$, (Phenomenex, Torrance, CA, USA) with flow rate of $0.2 \mathrm{ml} \mathrm{min}^{-1}$ using gradient solvents containing $5 \mathrm{mM} \mathrm{CH}_{3} \mathrm{COONH}_{4}$ (A-n-hexane:2-propanol:water, 43:57:1 (v/v/v) and B-n-hexane:2-propanol:water, 43:57:10 (v/v/v)). Tetra-myristoyl-cardiolipin (Avanti Polar Lipids) was used as an internal MS standard. Oxygenated cardiolipin was analysed by LC/MS as described ${ }^{66}$. Briefly, to prevent lipid oxidation, chromatography was performed under $\mathrm{N}_{2}$ on diethylenetriaminepentaacetic acid (DTPA)-treated silica plates $(5 \times 5 \mathrm{~cm}$, Whatman). LC/MS in negative mode was performed using a Dionex UltimateTM 3,000 RSLCnano system coupled online Q-Exactive hybrid quadrupole-orbitrap mass spectrometer (ThermoFisher Scientific, San Jose, CA, USA) using a C8 column (Luna $3 \mu \mathrm{m}, 100 \AA ̊, 150 \times 2 \mathrm{~mm}$, Phenomenex, Torrance, CA, USA) with flow rate $0.15 \mathrm{ml} \mathrm{min}^{-1}$ using an isocratic solvent system consisting of 2-propanol:water:triethylamine:acetic acid, 45:5:0.25:0.25 (v/v). The resolution was set up at 140,000, which corresponds to 5 p.p.m. in $\mathrm{m} / \mathrm{z}$ measurement error. Tetra-myristoyl-cardiolipin (Avanti polar lipids, Alabaster, AL, USA) was used as an internal MS standard. In addition, Tetra-linoleylcardiolipin was also utilized as a reference standard to build calibration curves employed for quantitation of all cardiolipin species.

RNA isolation and qRT-PCR. Lungs were dissociated in TRIzol (Life Technologies) using a high-speed homogenizer. RNA was isolated using RNeasy kit (Qiagen) and treated with RNase-free DNase (Qiagen). cDNA was synthesized using High Capacity cDNA Reverse Transcription kit (Life technologies) according to the manufacturer's instructions. Real-time quantitative PCR was performed using validated TaqMan Gene Expression primer and probe sets (Life Technologies) according to the manufacturer's instructions. Results were analysed using the SDS 2.2.2 software. mRNA expression was calculated using the $2^{-\Delta \mathrm{Ct}}$ method using hprt1 as internal reference control.

Transfection and reporter assay. Cells were transfected with either IL-10-luciferase reporter construct (Adgene) or PPRE-luciferase reporter construct (Qiagen, USA, Cat \# CCS-3026L), using Amaxa Mouse Macrophage Nucleofector Kit from Lonza (Cat \# VPA-1009) following manufacturer's instructions. Reporter assay was carried out $24 \mathrm{~h}$ post-transfection using dual luciferase assay kit (Promega, Cat \# E1910) following manufacturer's protocol. Briefly, cells were washed with PBS followed by lysis with 1X passive lysis buffer. The lysate was cleared by brief centrifugation and firefly luciferase reporter activity in the clear supernatant was measured using a luminometer (Veritas, Promega). Renilla luciferase activity in the same lysate was used to assess transfection efficiency.

Immunoprecipitation. Immunoprecipitation assay was performed using Pierce Crosslink Immunoprecipitation Kit (Cat \# 26147) following supplier's instructions. Briefly, desired antibodies (10 $\mu \mathrm{g}$ per IP) were adsorbed onto protein A/G agarose and crosslinked. Cells were washed with cold PBS and lysed. Cell lysates were cleared by centrifugation and supernatants were collected. Pre-clearing of cell lysates was done by mixing with washed agarose slurry and incubating for $1 \mathrm{~h}$ on a rocker at $4{ }^{\circ} \mathrm{C}$. Protein $\mathrm{A} / \mathrm{G}$ agarose beads were removed by centrifugation and supernatants were transferred to fresh tubes. Protein concentration was determined by Bradford assay. Six hundred microgram of total protein was incubated with $10 \mu \mathrm{g}$ of antibody-crosslinked protein $\mathrm{A} / \mathrm{G}$ agarose overnight on a rocker at $4{ }^{\circ} \mathrm{C}$. After multiple washes, the antigen was eluted with $50 \mu$ of elution buffer and analysed on SDS-PAGE (polyacrylamide gel electrophoresis) gel.
ChIP assay. ChIP assays were performed using Pierce AgaroseChIP kit (Thermo scientific, Cat \# 26156) following manufacturer's instructions. Briefly, thioglycollate-elicited peritoneal macrophages treated with either LPS alone or in combination with cardiolipin were crosslinked using formaldehyde and harvested by scraping. The cell pellet was resuspended in lysis buffer provided with the kit, and chromatin was immunoprecipitated using antibodies against PPAR $\gamma$, NCOR, HDAC3, p300 or NF-אB (p65). Immunoprecipitated genomic DNA and specific primers (FP $5^{\prime}$ TATCGGACGTTCAACCCAGG 3'; RP $5^{\prime}$ GGCCCTCATCTGTGGATTCC $3^{\prime}$ ) were used to amplify the region containing the PPRE at -349 bp of the IL-10 promoter. Different primers (FP 5' CTTCAGCCACTTCCTCCAAG 3'; RP 5' CATCCATGGGGGAGAA CTTA $3^{\prime}$ ) were used to amplify the region containing the NF- $\mathrm{KB}$ response element at $-513 \mathrm{bp}$ in the TNF promoter.

Western blot analysis. Cell lysates were prepared by harvesting cells in cell lysis buffer (Cell Signaling Technology, Cat \# 9830S) supplemented with protease inhibitors (Thermo scientific, Cat \# 87786). After clearing the lysate by centrifugation, supernatants were collected, and total protein content was measured by Bradford assay. Thirty microgram of total protein was resolved on a $10 \%$ SDS-PAGE gel and the proteins were transferred to a polyvinylidenedifluoride membrane. The blot was probed with the specific primary antibody followed by HRP-conjugated secondary antibody (Pierce) and developed using SuperSignal West Femtochemiluminescence substrate (Pierce, Cat \# 34095).

siRNA-mediated knockdown of gene expression. siRNAs specific to PPAR $\gamma$ were purchased from Dharmacon, USA (cat \# L-040712-00-0005) while the scrambled siRNAmix was from Santa Cruz Biotechnology, USA. Mice were treated with LPS once over three consecutive days ( $10 \mu \mathrm{g}$ each day) and lung MDSCs were isolated 1 day after last treatment and used for transfection ( $10^{6} \mathrm{cells} /$ transfection). Cells were transfected with either specific siRNA or scrambled siRNA using Amaxa Mouse Macrophage Nucleofector Kit from Lonza following manufacturer's instructions. Post-transfection, cells were maintained at $37^{\circ} \mathrm{C}$ in the presence of $5 \% \mathrm{CO}_{2}$. The efficiency of gene knockdown was verified by qRT-PCR $24 \mathrm{~h}$ posttransfection.

Lung histology. Excised lungs were stored in Safefix II (Fischer Scientific, Pittsburgh, PA) for $48 \mathrm{~h}$ for fixation and then transferred to $70 \%$ ethanol until paraffin-embedding for H\&E staining. For staining of OxPhos, lung sections were stained with antibody against oxidized phospholipids (clone EO6, Cat\# 330001S from Avanti Polar Lipids Inc. used at 1:100 dilution) in combination with Vector M.O.M immunodetection kit (Vector Laboratories, Cat\# PK-2200). DAB peroxidase substrate was used to detect positive EO6 staining (brown colour). The slides were then counterstained with hematoxylin, dehydrated, cleared and mounted.

Assay of secreted cytokines. Lungs were homogenized in a buffer containing $50 \mathrm{mM}$ Tris $\cdot \mathrm{HCl}, \mathrm{pH} 7.4,150 \mathrm{mM} \mathrm{NaCl}, 0.02 \%$ Tween 20 , and Complete Mini, EDTA-free protease inhibitor (Roche Applied Science) and debris-free supernatant was used for cytokine measurement. Secreted cytokines in mouse lung extracts were measured by magnetic bead-based assay (Bio-Rad Laboratories, Hercules, CA) according to the manufacturer's instructions. Data was acquired and analysed using Luminex automated system (BioRad Laboratories, Hercules, CA, USA). The level of IL-10 protein in supernatants of ex vivo cultures was measured using a DuoSet Elisa kit (R\&D Systems, Cat \# DY417) according to the manufacturer's protocol.

Measurement of albumin in BAL. Amount of albumin leak in BAL was measured using mouse albumin-specific ELISA kit (Abcam, Cat \# ab108792) following the manufacturer's instructions.

MPO assay. MPO assay was performed as described previously ${ }^{16}$. Briefly, a $2.5 \mathrm{mg} \mathrm{ml}^{-1}$ solution of O-Dianidinedihydrochloride (Cat \# D3252) (Sigma, USA) in $0.1 \%$ BSA/PBS was mixed with another solution of $0.1 \% \mathrm{H}_{2} \mathrm{O}_{2}$ prepared in $0.1 \%$ BSA/PBS. Two hundred microlitre of this solution was added to $50 \mu \mathrm{l}$ of standard or sample (BAL) and incubated for $15 \mathrm{~min}$ at $37^{\circ} \mathrm{C}$. The reaction was stopped by adding $50 \mu \mathrm{l}$ of $2 \%$ sodium azide (Sigma-Aldrich, USA). The colour developed was read at $450 \mathrm{~nm}$ wavelength of light.

Flow cytometry. Single-cell suspensions stained with combinations of antibodies listed above were acquired on FACS Aria (BD Immunocytometry Systems) and the data were analysed using FlowJo software (Tree Star).

Site-directed mutagenesis. K107 and K395 SUMOylation sites in PPAR $\gamma$ were mutated to arginine using QuikChange II XL Site-Directed Mutagenesis Kit (Cat \# 200521) from Agilent Technologies following manufacturer's instructions. Briefly, PPAR $\gamma$ overexpression construct (purchased from Addgene; Cat \# 8895) was used as template in a PCR containing the primers harboring the desired 
mutations. Of note, the expression construct encodes $\operatorname{PPAR} \gamma 2$, which is an isoform of PPAR $\gamma 1$, with the former containing 30 additional amino acids in the $\mathrm{N}$-terminus in the human protein. PPAR $\gamma 1$ is expressed in many cell types including hematopoietic cells while PPAR $\gamma 2$ is expressed in adipose tissue although expression can be induced in other tissues by high-fat diet ${ }^{57}$. Importantly, the two proteins PPAR $\gamma 1$ and PPAR $\gamma 2$ have similar functions in transfection experiments ${ }^{67}$. Following PCR amplification, the methylated template strands were removed by digestion with $\mathrm{DpnI}$ and the undigested constructs containing the desired mutations were used for transforming transformation-competent E. coli cells. The plasmids were purified and used for transfecting RAW 264.7 cells (ATCC, Cat \# ATCC TIB-71)

Statistical analysis. Results shown are mean values \pm s.d. One-way ANOVA with Bonferroni post hoc test was used for multiple pairwise comparisons. Student's unpaired two-tailed $t$ test was used for comparisons involving two groups. Differences between groups were considered significant when $P \leq 0.05$ Kaplan-Meier survival curves are derived from two experiments. The overall $P$ value was calculated using log-rank test. All statistical analyses were performed using GraphPad Prism 5 software (La Jolla, CA, USA).

Data availability. The data supporting the conclusions of the study are available from the authors upon reasonable request.

\section{References}

1. Nathan, C. \& Ding, A. Nonresolving inflammation. Cell 140, 871-882 (2010).

2. Bhattacharya, J. \& Matthay, M. A. Regulation and repair of the alveolarcapillary barrier in acute lung injury. Annu. Rev. Physiol. 75, 593-615 (2013).

3. Mizgerd, J. P. Acute lower respiratory tract infection. N. Engl. J. Med. 358, 716-727 (2008).

4. Levy, B. D. \& Serhan, C. N. Resolution of acute inflammation in the lung. Annu. Rev. Physiol. 76, 467-492 (2014).

5. Buckley, C. D., Gilroy, D. W., Serhan, C. N., Stockinger, B. \& Tak, P. P. The resolution of inflammation. Nat. Rev. Immunol. 13, 59-66 (2013).

6. Leslie, M. Inflammation's stop signals. Science 347, 18-21 (2015).

7. Barton, G. M. A calculated response: control of inflammation by the innate immune system. J. Clin. Invest. 118, 413-420 (2008).

8. Soehnlein, O. \& Lindbom, L. Phagocyte partnership during the onset and resolution of inflammation. Nat. Rev. Immunol. 10, 427-439 (2010).

9. Ortega-Gomez, A., Perretti, M. \& Soehnlein, O. Resolution of inflammation: an integrated view. EMBO Mol. Med. 5, 661-674 (2013).

10. Abraham, E. Neutrophils and acute lung injury. Crit. Care Med. 31, S195-S199 (2003).

11. Balamayooran, G., Batra, S., Fessler, M. B., Happel, K. I. \& Jeyaseelan, S. Mechanisms of neutrophil accumulation in the lungs against bacteria. Am. J. Respir. Cell Mol. Biol. 43, 5-16 (2010).

12. Lee, W. L. \& Downey, G. P. Neutrophil activation and acute lung injury. Curr. Opin. Crit. Care 7, 1-7 (2001)

13. Jones, R. N. Microbial etiologies of hospital-acquired bacterial pneumonia and ventilator-associated bacterial pneumonia. Clin. Infect. Dis. 51, S81-S87 (2010).

14. Arora, M. et al. TLR4/MyD88-induced CD11b ${ }^{+} \mathrm{Gr}-1^{\text {int }} \mathrm{F} 4 / 80^{+}$non-migratory myeloid cells suppress Th2 effector function in the lung. Mucosal Immunol. 3, 578-593 (2010).

15. Condamine, T. \& Gabrilovich, D. I. Molecular mechanisms regulating myeloidderived suppressor cell differentiation and function. Trends Immunol. 32, 19-25 (2011).

16. Poe, S. L. et al. STAT1-regulated lung MDSC-like cells produce IL-10 and efferocytose apoptotic neutrophils with relevance in resolution of bacterial pneumonia. Mucosal Immunol. 6, 189-199 (2013).

17. Ray, N. B. et al. Dynamic regulation of cardiolipin by the lipid pump Atp8b1 determines the severity of lung injury in experimental pneumonia. Nat. Med. 16, 1120-1127 (2010).

18. Kagan, V. E. et al. Cytochrome c acts as a cardiolipin oxygenase required for release of proapoptotic factors. Nat. Chem. Biol. 1, 223-232 (2005).

19. Krysko, D. V. et al. Emerging role of damage-associated molecular patterns derived from mitochondria in inflammation. Trends Immunol. 32, 157-164 (2011).

20. Claypool, S. M. \& Koehler, C. M. The complexity of cardiolipin in health and disease. Trends Biochem. Sci. 37, 32-41 (2012).

21. Pascual, G. et al. A SUMOylation-dependent pathway mediates transrepression of inflammatory response genes by PPAR-gamma. Nature 437, 759-763 (2005).

22. Balasubramanian, K. et al. Dichotomous roles for externalized cardiolipin in extracellular signaling: promotion of phagocytosis and attenuation of innate immunity. Sci. Signal. 8, ra95 (2015).
23. Ray, A., Chakraborty, K. \& Ray, P. Immunosuppressive MDSCs induced by TLR signaling during infection and role in resolution of inflammation. Front Cell Infect. Microbiol. 3, 52 (2013).

24. Saraiva, M. \& O'Garra, A. The regulation of IL-10 production by immune cells Nat. Rev. Immunol. 10, 170-181 (2010).

25. Wahli, W. \& Michalik, L. PPARs at the crossroads of lipid signaling and inflammation. Trends Endocrinol. Metab. 23, 351-363 (2012).

26. Poulsen, L., Siersbaek, M. \& Mandrup, S. PPARs: fatty acid sensors controlling metabolism. Semin. Cell Dev. Biol. 23, 631-639 (2012).

27. Katayama, K. et al. A novel PPAR gamma gene therapy to control inflammation associated with inflammatory bowel disease in a murine model Gastroenterology 124, 1315-1324 (2003).

28. Are, A. et al. Enterococcus faecalis from newborn babies regulate endogenous PPARgamma activity and IL-10 levels in colonic epithelial cells. Proc. Natl Acad. Sci. USA 105, 1943-1948 (2008).

29. Ghisletti, S. et al. Parallel SUMOylation-dependent pathways mediate gene- and signal-specific transrepression by LXRs and PPARgamma. Mol. Cell 25, 57-70 (2007).

30. Wadosky, K. M. \& Willis, M. S. The story so far: post-translational regulation of peroxisome proliferator-activated receptors by ubiquitination and SUMOylation. Am. J. Physiol. Heart Circ. Physiol. 302, H515-H526 (2012).

31. van Beekum, O., Fleskens, V. \& Kalkhoven, E. Posttranslational modifications of PPAR-gamma: fine-tuning the metabolic master regulator. Obesity 17, 213-219 (2009).

32. Floyd, Z. E. \& Stephens, J. M. Control of peroxisome proliferator-activated receptor gamma2 stability and activity by SUMOylation. Obes. Res. 12, 921-928 (2004).

33. Ohshima, T., Koga, H. \& Shimotohno, K. Transcriptional activity of peroxisome proliferator-activated receptor gamma is modulated by SUMO-1 modification. J. Biol. Chem. 279, 29551-29557 (2004).

34. Rangwala, S. M. et al. Genetic modulation of PPARgamma phosphorylation regulates insulin sensitivity. Dev. Cell 5, 657-663 (2003).

35. Yamashita, D. et al. The transactivating function of peroxisome proliferatoractivated receptor gamma is negatively regulated by SUMO conjugation in the amino-terminal domain. Genes Cells 9, 1017-1029 (2004).

36. Tsukahara, T. et al. Phospholipase D2-dependent inhibition of the nuclear hormone receptor PPARgamma by cyclic phosphatidic acid. Mol. Cell 39, 421-432 (2010).

37. Davie, J. R. Inhibition of histone deacetylase activity by butyrate. J. Nutr. 133, 2485S-2493S (2003).

38. Gabrilovich, D. I. \& Nagaraj, S. Myeloid-derived suppressor cells as regulators of the immune system. Nat. Rev. Immunol. 9, 162-174 (2009).

39. Ostrand-Rosenberg, S. \& Sinha, P. Myeloid-derived suppressor cells: linking inflammation and cancer. J. Immunol. 182, 4499-4506 (2009).

40. Iyer, S. S. et al. Mitochondrial cardiolipin is required for Nlrp3 inflammasome activation. Immunity 39, 311-323 (2013)

41. Greenhill, C. J. et al. Interleukin-10 regulates the inflammasome-driven augmentation of inflammatory arthritis and joint destruction. Arthritis Res. Ther. 16, 419 (2014).

42. Zhang, J., Fu, S., Sun, S., Li, Z. \& Guo, B. Inflammasome activation has an important role in the development of spontaneous colitis. Mucosal Immunol. 7, 1139-1150 (2014).

43. Saraiva, M. et al. Interleukin-10 production by Th1 cells requires interleukin12 -induced STAT4 transcription factor and ERK MAP kinase activation by high antigen dose. Immunity 31, 209-219 (2009).

44. Brightbill, H. D., Plevy, S. E., Modlin, R. L. \& Smale, S. T. A prominent role for Sp1 during lipopolysaccharide-mediated induction of the IL-10 promoter in macrophages. J. Immunol. 164, 1940-1951 (2000).

45. Xu, J. et al. c-Maf regulates IL-10 expression during Th17 polarization. J. Immunol. 182, 6226-6236 (2009).

46. Pot, C. et al. Cutting edge: IL-27 induces the transcription factor c-Maf, cytokine IL-21, and the costimulatory receptor ICOS that coordinately act together to promote differentiation of IL-10-producing Tr1 cells. J. Immunol. 183, 797-801 (2009).

47. Shoemaker, J., Saraiva, M. \& O'Garra, A. GATA-3 directly remodels the IL-10 locus independently of IL-4 in CD4 + T cells. J. Immunol. 176, 3470-3479 (2006).

48. Chang, H. D. et al. Expression of IL-10 in Th memory lymphocytes is conditional on IL-12 or IL-4, unless the IL-10 gene is imprinted by GATA-3. Eur. J. Immunol. 37, 807-817 (2007).

49. Ananieva, O. et al. The kinases MSK1 and MSK2 act as negative regulators of Toll-like receptor signaling. Nat. Immunol. 9, 1028-1036 (2008).

50. Mori, N. \& Prager, D. Activation of the interleukin-10 gene in the human T lymphoma line HuT 78: identification and characterization of NF-kappa B binding sites in the regulatory region of the interleukin-10 gene. Eur. J. Haematol. 59, 162-170 (1997). 
51. Cao, S., Zhang, X., Edwards, J. P. \& Mosser, D. M. NF-kappaB1 (p50) homodimers differentially regulate pro- and anti-inflammatory cytokines in macrophages. J. Biol. Chem. 281, 26041-26050 (2006).

52. Damm, K., Thompson, C. C. \& Evans, R. M. Protein encoded by v-erbA functions as a thyroid-hormone receptor antagonist. Nature 339, 593-597 (1989).

53. Sap, J., Munoz, A., Schmitt, J., Stunnenberg, H. \& Vennstrom, B. Repression of transcription mediated at a thyroid hormone response element by the $v$-erb-A oncogene product. Nature 340, 242-244 (1989).

54. Glass, C. K. \& Ogawa, S. Combinatorial roles of nuclear receptors in inflammation and immunity. Nat. Rev. Immunol. 6, 44-55 (2006).

55. Glass, C. K. \& Saijo, K. Nuclear receptor transrepression pathways that regulate inflammation in macrophages and T cells. Nat. Rev. Immunol. 10, 365-376 (2010).

56. Greenberger, M. J. et al. Neutralization of IL-10 increases survival in a murine model of Klebsiella pneumonia. J. Immunol. 155, 722-729 (1995).

57. Ahmadian, M. et al. PPARgamma signaling and metabolism: the good, the bad and the future. Nat. Med. 19, 557-566 (2013).

58. Kersten, S., Desvergne, B. \& Wahli, W. Roles of PPARs in health and disease. Nature 405, 421-424 (2000).

59. Semple, R. K., Chatterjee, V. K. \& O’Rahilly, S. PPAR gamma and human metabolic disease. J. Clin. Invest. 116, 581-589 (2006).

60. Anbalagan, M., Huderson, B., Murphy, L. \& Rowan, B. G. Post-translational modifications of nuclear receptors and human disease. Nucl. Recept. Signal 10, e001 (2012).

61. Smith, P. M. et al. The microbial metabolites, short-chain fatty acids, regulate colonic Treg cell homeostasis. Science 341, 569-573 (2013).

62. Tan, J. et al. The role of short-chain fatty acids in health and disease. Adv. Immunol. 121, 91-119 (2014).

63. Liang, X. et al. Sodium butyrate protects against severe burn-induced remote acute lung injury in rats. PLoS ONE 8, e68786 (2013).

64. Folch, J., Lees, M. \& Sloane Stanley, G. H. A simple method for the isolation and purification of total lipides from animal tissues. J. Biol. Chem. 226, 497-509 (1957).

65. Bottcher, C. J. F., van Gent, C. M. \& Pries, C. A rapid and sensitive sub-micro phosphorus determination. Anal. Chim. Acta 24, 203-204 (1961).

66. Tyurina, Y. Y. et al. A mitochondrial pathway for biosynthesis of lipid mediators. Nat. Chem. 6, 542-552 (2014).

67. Mueller, E. et al. Genetic analysis of adipogenesis through peroxisome proliferator-activated receptor gamma isoforms. J. Biol. Chem. 277, 41925-41930 (2002)

\section{Acknowledgements}

This work was supported by NIH grants PO1 HL114453 (R.K.M., P.R. and V.E.K), RO1 AI100012 (P.R.), HL122307 (P.R.), RO1 AI048927 and PO1 106684 (A.R.).

\section{Author contributions}

K.C. designed and performed experiments, analysed data and wrote the manuscript. M.R. performed experiments and analysed data. M.R. performed cytokine analysis. B.B.C. performed confocal microscopy and analysed data. C.M. performed lung histology and immunohistochemistry and analysed data. Y.Y.T. performed mass spectroscopy experiments and analysed data. A.K. and T.B.O. performed cell sorting and analysed flow cytometry data. C.M. and R.H. performed mouse surgeries. J.S.L. helped in in vivo model and provided helpful comments. C.M.S. and S.W. assisted in confocal microscopy and analysed data. R.K.M. analysed data and provided helpful comments. V.E.K. assisted with mass spectrometry experiments, analysed data and provided helpful comments. A.R. designed experiments, analysed data and wrote the manuscript. P.R. conceived of the study, designed experiments, analysed data and wrote the manuscript.

\section{Additional information}

Supplementary Information accompanies this paper at http://www.nature.com/ naturecommunications

Competing financial interests: The authors declare no competing financial interests.

Reprints and permission information is available online at http://npg.nature.com/ reprintsandpermissions/

How to cite this article: Chakraborty, K. et al. The mito-DAMP cardiolipin blocks IL-10 production causing persistent inflammation during bacterial pneumonia. Nat. Commun. 8, 13944 doi: $10.1038 /$ ncomms13944 (2017)

Publisher's note: Springer Nature remains neutral with regard to jurisdictional claims in published maps and institutional affiliations.

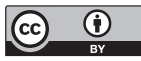

This work is licensed under a Creative Commons Attribution 4.0 International License. The images or other third party material in this article are included in the article's Creative Commons license, unless indicated otherwise in the credit line; if the material is not included under the Creative Commons license, users will need to obtain permission from the license holder to reproduce the material. To view a copy of this license, visit http://creativecommons.org/licenses/by/4.0/

(C) The Author(s) 2017 